\title{
Product Approximations for a Class of Quantum Anharmonic Oscillators
}

\author{
Wesley P. Petersen* and Pierre-A. Vuillermot** \\ Seminar for Applied Mathematics, ETHZ, Zurich, Switzerland* \\ UMR-CNRS 7502, Institut Élie Cartan de Lorraine, France**
}

\begin{abstract}
In this article we investigate analytically and numerically a class of non-autonomous Schrödinger equations in one space dimension describing the dynamics of quantum anharmonic oscillators driven by timedependent quartic interactions. We do so within a suitably constructed Faedo-Galerkin scheme by analyzing several product approximations for their solutions, which involve various exponential operator splittings. Our main objective is to study the convergence rates and the accuracy of such approximations, among which there are extensions of the Trotter-Kato product formula and several other variants. Crucial to our analysis is the knowledge of the lowest energy level and of the corresponding eigensolution to the associated time-independent problem, which we also compute within the very same framework.
\end{abstract}

\section{Introduction and Outline}

Our primary purpose in this article is to investigate from an analytical and a numerical point of view the class of one-dimensional non-autonomous Schrödinger initial-value problems given by

$$
\begin{aligned}
i \frac{\partial u(x, t)}{\partial t} & =\left(-\frac{1}{2} \frac{\partial^{2}}{\partial x^{2}}+\frac{1}{2} x^{2}+\lambda a(t) x^{4}\right) u(x, t), \quad(x, t) \in \mathbb{R} \times(0, T], \\
u(x, 0) & =v(x), \quad x \in \mathbb{R}
\end{aligned}
$$

where $\lambda>0$ and where $a:[0, T] \mapsto \mathbb{R}^{+}$is a twice continuously differentiable and positive amplitude function, with $T \in(0,+\infty)$ arbitrary. Problems of the form (1) describe the dynamics of quantum anharmonic oscillators driven by time-dependent quartic interactions, whose autonomous version

$$
\begin{aligned}
i \frac{\partial u(x, t)}{\partial t} & =\left(-\frac{1}{2} \frac{\partial^{2}}{\partial x^{2}}+\frac{1}{2} x^{2}+\lambda x^{4}\right) u(x, t), \quad(x, t) \in \mathbb{R} \times(0, T], \\
u(x, 0) & =v(x), \quad x \in \mathbb{R}
\end{aligned}
$$


has triggered over the years some interest in theoretical physics as a simple model in field theory (see, for instance, [4] and [24]). From a mathematical point of view, (1) may be considered as a very particular example of evolution problems of the form

$$
\begin{aligned}
i \frac{d u(t)}{d t} & =H(t) u(t), \quad t \in(0, T], \\
u(0) & =v
\end{aligned}
$$

defined in a complex Hilbert space $\mathcal{H}$, where the $H(t)$ 's are unbounded selfadjoint operators on some time-dependent and dense domains $\mathcal{D}(H(t)) \subset \mathcal{H}$. In the case of (1) the reference space is the usual complex Lebesgue space $\mathcal{H}=$ $L^{2}(\mathbb{R}, \mathbb{C})$ endowed with the inner product

$$
(f, g)_{2}:=\int_{\mathbb{R}} d x f(x) \bar{g}(x),
$$

and the $H(t)$ 's are self-adjoint realizations of the formal expressions

$$
H(t):=-\frac{1}{2} \frac{\partial^{2}}{\partial x^{2}}+\frac{x^{2}}{2}+\lambda a(t) x^{4} .
$$

There are many ways to compute the solution to evolution equations of the form (3). For instance, writing $\mathcal{L}(\mathcal{H})$ for the algebra of all linear bounded operators on $\mathcal{H}$, and assuming there is a unitary evolution system $U_{H}(t, s)_{s, t \in[0, T]}$ on $\mathcal{H}$ such that

$$
u(t):=U_{H}(t, 0) v
$$

solves (3) in a suitable sense, we may ask whether there exist one-parameter families of functions $F_{t}: \mathbb{R}^{+} \mapsto \mathcal{L}(\mathcal{H})$ such that formulae of the form

$$
U_{H}(t, s)=\lim _{n \rightarrow+\infty} \prod_{\gamma=n-1}^{0} F_{s+\frac{\gamma}{n}(t-s)}\left(\frac{t-s}{n}\right)
$$

hold true in one of the various topologies of $\mathcal{L}(\mathcal{H})$ for all $s, t \in[0, T]$ with $t \geq s$. Formulae such as (6) indeed constitute the theoretical basis of numerical algorithms intended to compute solutions to various evolution problems, a recurrent theme that was already thoroughly discussed in [9] in the context of autonomous evolution equations.

In the sequel we write $h:=\frac{t-s}{n}$ for the uniform time step so that (6) takes the equivalent form

$$
U_{H}(t, s)=\lim _{n \rightarrow+\infty} \prod_{\gamma=n-1}^{0} F_{s+\gamma h}(h),
$$

although we also allow the time variable to become large while keeping $h$ fixed in some numerical examples of Section 3. Product approximations such as (7) 
were in fact proved in [27] for a large class of $F_{t}$ 's by using an adaptation of the methods developed in [26] and [28] for the investigation of parabolic evolution equations, and were illustrated there by two examples in quantum mechanics.

In this article we consider more refined approximating functions than those introduced in [27], which we require to take values in the unitary group of $\mathcal{L}(\mathcal{H})$ for reasons which we explain further below. More specifically we investigate a situation that pertains to problems of the form (1), namely, one where more information is available regarding the structure of the $H(t)$ 's aside from their self-adjointness, such as a typical decomposition of the form

$$
H(t)=H_{0}+V(t)
$$

with $H_{0}$ and $V(t)$ both self-adjoint operators on $\mathcal{H}$ and $H_{0}$ independent of time. In such a case we can indeed think of many more concrete choices for $F_{t}$ which are not tractable by the method set forth in [27]. For instance we can consider

$$
F_{t}(\tau)=\exp \left[-i \tau H_{0}\right] \exp [-i \tau V(t)],
$$

in which case the corresponding approximation reads

$$
U_{H}(t, s)=\lim _{n \rightarrow+\infty} \prod_{\gamma=n-1}^{0} \exp \left[-i h H_{0}\right] \exp [-i h V(s+\gamma h)] .
$$

We remark that (10) represents a formal generalization of the usual Trotter-Kato formula

$$
\exp \left[-i(t-s)\left(H_{0}+V\right)\right]=\lim _{n \rightarrow+\infty}\left(\exp \left[-i h H_{0}\right] \exp [-i h V]\right)^{n}
$$

which is known to hold in the strong operator topology of $\mathcal{L}(\mathcal{H})$ if $H_{0}+V$ is essentially self-adjoint on the domain $\mathcal{D}\left(H_{0}\right) \cap \mathcal{D}(V)$, and which has been generalized in various directions over the years (see, for instance, [7], [8], [13], [14], [25] and also [10], [20], [24] and more recently [12] for a comprehensive review of these and related works). But we can also consider

$$
F_{t}(\tau)=\exp \left[-i \tau H_{0}\right] \exp [-i \tau V(t)] \exp \left[\frac{\tau^{2}}{2}\left[H_{0}, V(t)\right]_{-}\right]
$$

provided the commutator $\left[H_{0}, V(t)\right]_{-}$makes sense as an anti-adjoint operator on some suitable domain in $\mathcal{H}$, or

$$
F_{t}(\tau)=\exp \left[-i \frac{\tau}{2} H_{0}\right] \exp [-i \tau V(t)] \exp \left[-i \frac{\tau}{2} H_{0}\right]
$$

among many other possible choices. In these two cases the corresponding approximations formally read

$$
\begin{aligned}
& U_{H}(t, s) \\
& =\lim _{n \rightarrow+\infty} \prod_{\gamma=n-1}^{0} \exp \left[-i h H_{0}\right] \exp [-i h V(s+\gamma h)] \exp \left[\frac{h^{2}}{2}\left[H_{0}, V(s+\gamma h)\right]_{-}\right]
\end{aligned}
$$


and

$$
U_{H}(t, s)=\lim _{n \rightarrow+\infty} \prod_{\gamma=n-1}^{0} \exp \left[-i \frac{h}{2} H_{0}\right] \exp [-i h V(s+\gamma h)] \exp \left[-i \frac{h}{2} H_{0}\right],
$$

respectively. The choice of (11) has its origins in the Baker-Campbell-Hausdorff formula of Lie group theory, as is the case for (12) which pertains to the construction of the symmetric splitting schemes introduced in [29] (see, for instance, Chapter 3 in [16]).

A further variant of the last two choices amounts to replacing everywhere the values $V(s+\gamma h)$ of the function $V$ at the subdivision points by the arithmetic averages

$$
W_{\gamma}(s, h):=\frac{1}{2}(V(s+\gamma h)+V(s+(\gamma+1) h))
$$

for each $\gamma \in\{0, \ldots, n-1\}$. Formulae (13) and (14) then become

$$
\begin{aligned}
& U_{H}(t, s) \\
& =\lim _{n \rightarrow+\infty} \prod_{\gamma=n-1}^{0} \exp \left[-i h H_{0}\right] \exp \left[-i h W_{\gamma}(s, h)\right] \exp \left[\frac{h^{2}}{2}\left[H_{0}, W_{\gamma}(s, h)\right]_{-}\right]
\end{aligned}
$$

and

$$
U_{H}(t, s)=\lim _{n \rightarrow+\infty} \prod_{\gamma=n-1}^{0} \exp \left[-i \frac{h}{2} H_{0}\right] \exp \left[-i h W_{\gamma}(s, h)\right] \exp \left[-i \frac{h}{2} H_{0}\right],
$$

respectively.

To the best of our knowledge and in contrast to the parabolic case that was investigated in [26] and [28], there exist as yet no proofs of the above product approximations for the solution to evolution problems of the form

$$
\begin{aligned}
i \frac{d u(t)}{d t} & =\left(H_{0}+V(t)\right) u(t), \quad t \in(0, T], \\
u(0) & =v
\end{aligned}
$$

when the operators $H_{0}$ and $V(t)$ are unbounded. This remark applies in particular to Problem (18) with the self-adjoint realizations of

$$
H_{0}:=-\frac{1}{2} \frac{\partial^{2}}{\partial x^{2}}+\frac{x^{2}}{2}
$$

and

$$
V(t):=\lambda a(t) x^{4},
$$

and thereby constitutes the point of departure of our investigation. Our goal is indeed to compute the solution to (18)-(20) using the example

$$
a(t)=1-\sin \omega t
$$


with $\omega>0$, as well as the solution and the energy levels of the corresponding autonomous problem (2), in such a way that we can test the validity of the above approximating formulae and simultaneously provide rates of convergence in each case. Accordingly, the remaining part of this article is organized as follows: in Section 2 we first endow $L^{2}(\mathbb{R}, \mathbb{C})$ with the orthonormal basis consisting of the Hermite functions $\mathrm{h}_{m}: \mathbb{R} \mapsto \mathbb{R}$ defined by

$$
\mathrm{h}_{m}(x):=\left(\pi^{\frac{1}{2}} 2^{m} m !\right)^{-\frac{1}{2}} e^{-\frac{x^{2}}{2}} \mathrm{H}_{m}(x)
$$

for every $m \in \mathbb{N}$, where the $\mathrm{H}_{m}$ 's are the Hermite polynomials

$$
\mathrm{H}_{m}(x)=(-1)^{m} e^{x^{2}} \frac{d^{m}}{d x^{m}} e^{-x^{2}}
$$

We then devise a Faedo-Galerkin scheme where for each $N \in \mathbb{N}^{+}$we denote by $E_{N}(\mathbb{R}, \mathbb{C})$ the finite-dimensional subspace of $L^{2}(\mathbb{R}, \mathbb{C})$ generated by $\left\{\mathrm{h}_{0}, \ldots, \mathrm{h}_{N-1}\right\}$, and consider there the truncated version of (18)

$$
\begin{aligned}
i \frac{d u_{N}(t)}{d t} & =\left(H_{0, N}+V_{N}(t)\right) u_{N}(t), \quad t \in(0, T], \\
u_{N}(0) & =v_{N}
\end{aligned}
$$

where $H_{0, N}$ and $V_{N}(t)$ are linear bounded self-adjoint operators corresponding to suitable restrictions of $(19)$ and $(20)$ to $E_{N}(\mathbb{R}, \mathbb{C})$, respectively, and where $v_{N}$ stands for the orthogonal projection of $v$ onto $E_{N}(\mathbb{R}, \mathbb{C})$. This allows us to prove the existence of unitary evolution operators $U_{N}(t, s)_{s, t \in[0, T]}$ such that

$$
u_{N}(t):=U_{N}(t, 0) v_{N}
$$

solves (23), while providing an approximation of the initial condition in (18) of the form

$$
v_{N}=\sum_{m=0}^{N-1} v_{m} \mathrm{~h}_{m}
$$

Such a result is indeed easy to obtain to the extent that the operators in (23) remain bounded. In Section 2 we also introduce the truncated versions of the right-hand sides of (10), (16) and (17), namely,

$$
u_{N, n}^{T K}(t):=\prod_{\gamma=n-1}^{0} \exp \left[-i h H_{0, N}\right] \exp \left[-i h V_{N}(s+\gamma h)\right] v_{N}
$$

$$
\begin{aligned}
& u_{N, n}^{B C H}(t) \\
& :=\prod_{\gamma=n-1}^{0} \exp \left[-i h H_{0, N}\right] \exp \left[-i h W_{N, \gamma}(s, h)\right] \exp \left[\frac{h^{2}}{2}\left[H_{0, N}, W_{N, \gamma}(s, h)\right]_{-}\right] v_{N}
\end{aligned}
$$


and

$$
u_{N, n}^{Y}(t):=\prod_{\gamma=n-1}^{0} \exp \left[-i \frac{h}{2} H_{0, N}\right] \exp \left[-i h W_{N, \gamma}(s, h)\right] \exp \left[-i \frac{h}{2} H_{0, N}\right] v_{N},
$$

where $T K, B C H$ and $Y$ stand for Trotter-Kato, Baker-Campbell-Hausdorff and Yoshida, respectively, and where

$$
W_{N, \gamma}(s, h):=\frac{1}{2}\left(V_{N}(s+\gamma h)+V_{N}(s+(\gamma+1) h)\right)
$$

is the truncated version of (15). We then prove that the speed of convergence of (27) and (28) toward (24) as $n \rightarrow+\infty$ is much faster than it would have been with the truncated versions of (13) and (14), respectively, thereby justifying the replacement of $V(s+\gamma h)$ by $W_{\gamma}(s, h)$ in those formulae. In contrast, we also show there that this replacement has no influence whatsoever regarding the convergence rate of (26).

Whereas the results of Section 2 are analytical and valid for problems of the form (1) with an arbitrary smooth amplitude function $a$, those of Section 3 are mainly numerical and concerned with the computation of the solutions to (2) and to the particular case of (1) given by

$$
\begin{aligned}
i \frac{\partial u(x, t)}{\partial t} & =\left(-\frac{1}{2} \frac{\partial^{2}}{\partial x^{2}}+\frac{1}{2} x^{2}+\lambda(1-\sin \omega t) x^{4}\right) u(x, t), \quad(x, t) \in \mathbb{R} \times(0, T] \\
u(x, 0) & =v(x), \quad x \in \mathbb{R} .
\end{aligned}
$$

The initial condition in (2) and (30) is chosen to be a solution to the eigenvalue problem

$$
\left(-\frac{1}{2} \frac{d^{2}}{d x^{2}}+\frac{1}{2} x^{2}+\lambda x^{4}\right) v(x)=E_{0} v(x)
$$

where $E_{0}$ is the lowest eigenvalue in the spectrum of this differential operator, so that both (2) and (30) are indeed intimately tied up since (31) is related to the stationary part of (2). More specifically, we first compute the solution to (2) by means of the formula

$$
u_{N}(t)=\exp \left[-i t\left(H_{0, N}+V_{N}\right)\right] v_{N}
$$

for various values of $N$ and $\lambda$ by means of MatLab's expm, and then compare the results with those obtained from the time-independent versions of (26), (27) and (28), namely,

$$
\begin{gathered}
u_{N, n}^{T K}(t)=\left(\exp \left[-i h H_{0, N}\right] \exp \left[-i h V_{N}\right]\right)^{n} v_{N}, \\
u_{N, n}^{B C H}(t)=\left(\exp \left[-i h H_{0, N}\right] \exp \left[-i h V_{N}\right] \exp \left[\frac{h^{2}}{2}\left[H_{0, N}, V_{N}\right]_{-}\right]\right)^{n} v_{N}
\end{gathered}
$$


and

$$
u_{N, n}^{Y}(t)=\left(\exp \left[-i \frac{h}{2} H_{0, N}\right] \exp \left[-i h V_{N}\right] \exp \left[-i \frac{h}{2} H_{0, N}\right]\right)^{n} v_{N}
$$

respectively, for $n$ sufficiently large. Quite independently as a control, we also compute the solution to (2) directly by using MatLab's ode45 (see, for instance, [11] for the origins of this method), and the comparison of that solution with those obtained by means of (32)-(35) reveals that our numerical results are very stable and accurate indeed. Of course all those computations first require the computation of, and very good approximations for, the smallest eigenvalue $E_{0}$ of the spectrum and for the corresponding eigenfunction $v$, which we get by means of MatLab's eigs and by a variational argument, respectively. At the same time our variational method also allows us to address the important issue of how large $N$ ought to be for our results to be reliable.

Quite independently again, we also compute some of the eigenvalues in

$$
\left(-\frac{1}{2} \frac{d^{2}}{d x^{2}}+\frac{1}{2} x^{2}+\lambda x^{4}\right) v(x)=E v(x)
$$

by using MatLab's eigs, with a particular emphasis on the lowest part of the spectrum $E_{0}$, thereby showing a good agreement with the analytical approximations.

We then proceed by computing the solution to (30) by using similar techniques, namely, first by means of

$$
u_{N, n}(t):=\prod_{\gamma=n-1}^{0} \exp \left[-i h\left(H_{0, N}+V_{N}(s+\gamma h)\right)\right] v_{N}
$$

and then with formulae (26), (27), (28) using MatLab's expm, and ultimately as a control by means of MatLab's ode45. The comparison of all those computations shows once more that our results are very stable and accurate, which in the end leads us to compare the numerical results of Section 3 with the analytical results of Section 2. Our conclusion is that all of the above product approximation formulae are indeed valid within the precision of our computations. Finally, we derive other estimates for $E_{0}$ and $v$ by means of a formal WKB approximation in the Appendix.

We conclude this introduction by pointing out that the methods of investigation set forth in this article are very different from other techniques which were developed in recent times by a variety of authors in various settings to deal with Schrödinger equations (see, for instance, [19] and some of the references therein). For example, in [19] the authors prove some error bounds on the basis of commutator estimates which involve unbounded operators from the outset, without ever considering Faedo-Galerkin approximations and time-dependent operators. The application of their results is thereby exclusively limited to the analysis of evolutionary Schrödinger equations with time-independent and bounded potentials. 


\section{Some Analytical Results}

We begin by recalling that the eigenvalue equation

$$
H_{0} \mathrm{~h}_{m}=\left(m+\frac{1}{2}\right) \mathrm{h}_{m}
$$

holds identically on $\mathbb{R}$ for every $m \in \mathbb{N}$, so that the space $E_{N}(\mathbb{R}, \mathbb{C})$ defined in the preceding section remains invariant under $H_{0}$. In the sequel we write $H_{0, N}$ for the restriction of $H_{0}$ to $E_{N}(\mathbb{R}, \mathbb{C})$, which obviously defines a linear bounded self-adjoint operator there. In order to get a restriction of the quartic term (20) to $E_{N}(\mathbb{R}, \mathbb{C})$ which enjoys similar properties, we first need the following result whose proof is omitted since it obtains from (22) and the recurrence relations for the $\mathrm{H}_{m}$ 's in a standard way (see, for instance, [1] for a statement of those recurrence relations):

Lemma 1. We have

$$
\begin{aligned}
& x^{4} \mathrm{~h}_{m}(x) \\
& =\frac{1}{4} \sqrt{m(m-1)(m-2)(m-3)} \mathrm{h}_{m-4}(x) \\
& +\frac{1}{2} \sqrt{m(m-1)}(2 m-1) \mathrm{h}_{m-2}(x) \\
& +\frac{3}{4}\left(2 m^{2}+2 m+1\right) \mathrm{h}_{m}(x) \\
& +\frac{1}{2} \sqrt{(m+1)(m+2)}(2 m+3) \mathrm{h}_{m+2}(x) \\
& +\frac{1}{4} \sqrt{(m+1)(m+2)(m+3)(m+4)} \mathrm{h}_{m+4}(x)
\end{aligned}
$$

for each $x \in \mathbb{R}$ and every $m \in \mathbb{N}$, provided we set $\mathrm{h}_{m^{\prime}}$ identically equal to zero whenever $m^{\prime}<0$.

Let us now introduce the shorthand notation

$$
\begin{aligned}
a_{m} & :=\frac{1}{4} \sqrt{m(m-1)(m-2)(m-3)} \\
b_{m} & :=\frac{1}{2} \sqrt{m(m-1)}(2 m-1) \\
c_{m} & :=\frac{3}{4}\left(2 m^{2}+2 m+1\right) \\
d_{m} & :=\frac{1}{2} \sqrt{(m+1)(m+2)}(2 m+3) \\
e_{m}: & :=\frac{1}{4} \sqrt{(m+1)(m+2)(m+3)(m+4)}
\end{aligned}
$$


for the coefficients in (37), so that we obtain

$$
\begin{aligned}
& \left(V(t) \mathrm{h}_{m}, \mathrm{~h}_{m^{\prime}}\right)_{2} \\
& =\lambda a(t)\left\{a_{m} \delta_{m-4, m^{\prime}}+b_{m} \delta_{m-2, m^{\prime}}+c_{m} \delta_{m, m^{\prime}}+d_{m} \delta_{m+2, m^{\prime}}+e_{m} \delta_{m+4, m^{\prime}}\right\}
\end{aligned}
$$

for all $m, m^{\prime} \in\{0, \ldots, N-1\}$ as a consequence of (20), Lemma 1 and the orthonormality of the $\mathrm{h}_{m}$ 's. This allows us to define $V_{N}(t)$ as the linear transformation in $E_{N}(\mathbb{R}, \mathbb{C})$ whose matrix elements relative to the basis $\left\{\mathrm{h}_{0}, \ldots, \mathrm{h}_{N-1}\right\}$ are given by $(38)$, and $V_{N}(t)$ is a self-adjoint operator there since the relations

$$
\left(V(t) \mathrm{h}_{m}, \mathrm{~h}_{m^{\prime}}\right)_{2}=\left(\mathrm{h}_{m}, V(t) \mathrm{h}_{m^{\prime}}\right)_{2}=\left(V(t) \mathrm{h}_{m^{\prime}}, \mathrm{h}_{m}\right)_{2}
$$

hold as a consequence of the fact that $a_{m+4}=e_{m}$ and $b_{m+2}=d_{m}$ for every $m$. For convenience we summarize these remarks in the following statement:

Lemma 2. The restrictions $H_{0, N}$ and $V_{N}(t)$ of $H_{0}$ and $V(t)$ defined above are both linear bounded self-adjoint operators in $E_{N}(\mathbb{R}, \mathbb{C})$.

Our main analytical result is then the following, in which $W_{N, \gamma}(s, h)$ is given by $(29)$ :

Theorem. (a) For every $N \in \mathbb{N}^{+}$let us define the operators $U_{N}(t, s)_{s, t \in[0, T]}$ in $E_{N}(\mathbb{R}, \mathbb{C})$ by

$$
\begin{aligned}
& U_{N}(t, s) \\
& :=\mathbb{I}_{N}+\sum_{\delta=1}^{+\infty}(-i)^{\delta} \int_{s}^{t} d \tau_{1} \ldots \int_{s}^{\tau_{\delta-1}} d \tau_{\delta}\left(H_{0, N}+V_{N}\left(\tau_{1}\right)\right) \ldots\left(H_{0, N}+V_{N}\left(\tau_{\delta}\right)\right),
\end{aligned}
$$

where $\mathbb{I}_{N}$ is the identity operator and $\tau_{0}=t$. Then each $U_{N}(t, s)$ is unitary and satisfies

$$
\begin{aligned}
U_{N}(t, t) & =\mathbb{I}_{N}, \\
U_{N}(t, s) & =U_{N}(t, r) U_{N}(r, s)
\end{aligned}
$$

for all $r, s, t \in[0, T]$. Moreover, the functions $u_{N}:[0, T] \mapsto E_{N}(\mathbb{R}, \mathbb{C})$ given by

$$
u_{N}(t)=U_{N}(t, 0) v_{N}
$$

are continuous, once continuously differentiable on $(0, T]$ and satisfy (23) identically, thereby providing a sequence of Faedo-Galerkin approximations to (18).

Furthermore, for every $N \in \mathbb{N}^{+}$and all $s, t \in[0, T]$ with $t \geq s$ the following statements hold: 
(b) We have

$$
\begin{aligned}
& U_{N}(t, s) \\
& =\prod_{\gamma=n-1}^{0} \exp \left[-i h H_{0, N}\right] \exp \left[-i h V_{N}(s+\gamma h)\right]+O_{N}(h) \\
& =\prod_{\gamma=n-1}^{0} \exp \left[-i h H_{0, N}\right] \exp \left[-i h W_{N, \gamma}(s, h)\right]+O_{N}(h)
\end{aligned}
$$

as $h \rightarrow 0_{+}$.

(c) We have

$$
\begin{aligned}
& U_{N}(t, s) \\
& =\prod_{\gamma=n-1}^{0} \exp \left[-i h H_{0, N}\right] \exp \left[-i h W_{N, \gamma}(s, h)\right] \exp \left[\frac{h^{2}}{2}\left[H_{0, N}, W_{N, \gamma}(s, h)\right]_{-}\right]+O_{N}\left(h^{2}\right) \\
& =\prod_{\gamma=n-1}^{0} \exp \left[-i \frac{h}{2} H_{0, N}\right] \exp \left[-i h W_{N, \gamma}(s, h)\right] \exp \left[-i \frac{h}{2} H_{0, N}\right]+O_{N}\left(h^{2}\right)
\end{aligned}
$$

as $h \rightarrow 0_{+}$.

Remarks. (1) Within the above framework Problem (2) corresponds to a time-independent potential $V_{N}$ in (23), in which case (41) and (42) all reduce to the well-known Lie-type formulae

$$
\begin{aligned}
& \exp \left[-i(t-s)\left(H_{0, N}+V_{N}\right)\right] \\
& =\left(\exp \left[-i h H_{0, N}\right] \exp \left[-i h V_{N}\right]\right)^{n}+O_{N}(h) \\
& =\left(\exp \left[-i h H_{0, N}\right] \exp \left[-i h V_{N}\right] \exp \left[\frac{h^{2}}{2}\left[H_{0, N}, V_{N}\right]_{-}\right]\right)^{n}+O_{N}\left(h^{2}\right) \\
& =\left(\exp \left[-i \frac{h}{2} H_{0, N}\right] \exp \left[-i h V_{N}\right] \exp \left[-i \frac{h}{2} H_{0, N}\right]\right)^{n}+O_{N}\left(h^{2}\right)
\end{aligned}
$$

as $h \rightarrow 0_{+}$. Therefore, the results of the preceding theorem generalize these relations to the non-autonomous case.

(2) Whereas the replacement of $V_{N}(s+\gamma h)$ by $W_{N, \gamma}(s, h)$ makes absolutely no difference regarding the rate of convergence in $(41)$, we stress the fact that it is essential for the estimates in (42) to hold: had we kept $V_{N}(s+\gamma h)$ instead of $W_{N, \gamma}(s, h)$ on the right-hand side of (42), we would have obtained the much slower convergence rate

$$
\begin{aligned}
& U_{N}(t, s) \\
& =\prod_{\gamma=n-1}^{0} \exp \left[-i h H_{0, N}\right] \exp \left[-i h V_{N}(s+\gamma h)\right] \exp \left[\frac{h^{2}}{2}\left[H_{0, N}, V_{N}(s+\gamma h)\right]_{-}\right]+O_{N}(h) \\
& =\prod_{\gamma=n-1}^{0} \exp \left[-i \frac{h}{2} H_{0, N}\right] \exp \left[-i h V_{N}(s+\gamma h)\right] \exp \left[-i \frac{h}{2} H_{0, N}\right]+O_{N}(h)
\end{aligned}
$$


as $h \rightarrow 0_{+}$. We will dwell a bit more on this further below.

(3) The rate of convergence in (42) does not change if we replace $W_{N, \gamma}(s, h)$ there by

$$
W_{N, \gamma}^{\prime}(s, h):=V_{N}\left(s+\gamma h+\frac{h}{2}\right),
$$

that is, by the value of the function $V_{N}$ at the mid-point between $s+\gamma h$ and $s+(\gamma+1) h$ for every $\gamma \in\{0, \ldots, n-1\}$, which amounts to replacing $W_{\gamma}(s, h)$ by

$$
W_{\gamma}^{\prime}(s, h):=V\left(s+\gamma h+\frac{h}{2}\right)
$$

in (15)-(17). The same observation holds if we exchange the rôles of $H_{0, N}$ and $W_{N, \gamma}(s, h)$ in the second line of $(42)$. The proofs of these assertions will be omitted, since they are similar to that given below for (42).

While Statement (a) corresponds to the so-called Dyson expansion and its most elementary consequences (see, for instance, Theorem X.69 in [24]), the proofs of (b) and (c) require some preliminary considerations. To this end and for every $\gamma \in\{0, \ldots, n-1\}$, we first define the operators

$$
\begin{aligned}
& U_{N, \gamma, n}(t, s) \\
& :=U_{N}(s+(\gamma+1) h, s+\gamma h) \\
& =\mathbb{I}_{N}+\sum_{\delta=1}^{+\infty}(-i)^{\delta} \int_{s+\gamma h}^{s+(\gamma+1) h} d \tau_{1} \ldots \int_{s+\gamma h}^{\tau_{\delta-1}} d \tau_{\delta}\left(H_{0, N}+V_{N}\left(\tau_{1}\right)\right) \ldots\left(H_{0, N}+V_{N}\left(\tau_{\delta}\right)\right)
\end{aligned}
$$

with $\tau_{0}=s+(\gamma+1) h$ according to (39). By virtue of the basic composition law (40) we get

$$
U_{N}(t, s)=\prod_{\gamma=n-1}^{0} U_{N, \gamma, n}(t, s) .
$$

Then, for each of the operators (45) we have:

Lemma 3. For all $n, N \in \mathbb{N}^{+}$and all $s, t \in[0, T]$ with $t \geq s$ the following statements hold:

(a) We have

$$
\begin{aligned}
& U_{N, \gamma, n}(t, s) \\
& =\exp \left[-i h H_{0, N}\right] \exp \left[-i h V_{N}(s+\gamma h)\right]+O_{N}\left(h^{2}\right) \\
& =\exp \left[-i h H_{0, N}\right] \exp \left[-i h W_{N, \gamma}(s, h)\right]+O_{N}\left(h^{2}\right)
\end{aligned}
$$

as $h \rightarrow 0_{+}$, uniformly in $\gamma \in\{0, \ldots, n-1\}$. 
(b) We have

$$
\begin{aligned}
& U_{N, \gamma, n}(t, s) \\
& =\exp \left[-i h H_{0, N}\right] \exp \left[-i h W_{N, \gamma}(s, h)\right] \exp \left[\frac{h^{2}}{2}\left[H_{0, N}, W_{N, \gamma}(s, h)\right]_{-}\right]+O_{N}\left(h^{3}\right) \\
& =\exp \left[-i \frac{h}{2} H_{0, N}\right] \exp \left[-i h W_{N, \gamma}(s, h)\right] \exp \left[-i \frac{h}{2} H_{0, N}\right]+O_{N}\left(h^{3}\right)
\end{aligned}
$$

as $h \rightarrow 0_{+}$, uniformly in $\gamma \in\{0, \ldots, n-1\}$.

Proof. In order to prove (47) we first split (45) as

$$
U_{N, \gamma, n}(t, s)=\mathbb{I}_{N}-i \int_{s+\gamma h}^{s+(\gamma+1) h} d \tau_{1}\left(H_{0, N}+V_{N}\left(\tau_{1}\right)\right)+\widehat{U}_{N, \gamma, n}(t, s)
$$

where

$$
\begin{aligned}
& \widehat{U}_{N, \gamma, n}(t, s) \\
& =\sum_{\delta=2}^{+\infty}(-i)^{\delta} \int_{s+\gamma h}^{s+(\gamma+1) h} d \tau_{1} \ldots \int_{s+\gamma h}^{\tau_{\delta-1}} d \tau_{\delta}\left(H_{0, N}+V_{N}\left(\tau_{1}\right)\right) \ldots\left(H_{0, N}+V_{N}\left(\tau_{\delta}\right)\right) .
\end{aligned}
$$

Furthermore we write $\|$.$\| for the usual matrix supremum-norm. Then we have$

$$
\left\|V_{N}(t)\right\| \leq c_{N}<+\infty
$$

for some $c_{N}$ uniformly in $t$ as a consequence of (38) and the definition of $V_{N}(t)$, so that

$$
\begin{aligned}
& \left\|\widehat{U}_{N, \gamma, n}(t, s)\right\| \\
& \leq \sum_{\delta=2}^{+\infty} \int_{s+\gamma h}^{s+(\gamma+1) h} d \tau_{1} \ldots \int_{s+\gamma h}^{\tau_{\delta-1}} d \tau_{\delta} \prod_{j=1}^{\delta}\left(\left\|H_{0, N}\right\|+\left\|V_{N}\left(\tau_{j}\right)\right\|\right) \\
& \leq \sum_{\delta=2}^{+\infty} \frac{h^{\delta}}{\delta !}\left(\left\|H_{0, N}\right\|+c_{N}\right)^{\delta}=O_{N}\left(h^{2}\right)
\end{aligned}
$$

as $h \rightarrow 0_{+}$. Moreover we also have

$$
\begin{aligned}
& \exp \left[-i h H_{0, N}\right] \exp \left[-i h V_{N}(s+\gamma h)\right] \\
& =\mathbb{I}_{N}-i h\left(H_{0, N}+V_{N}(s+\gamma h)\right)+O_{N}\left(h^{2}\right)
\end{aligned}
$$

by expanding each exponential by means of the exponential series. Consequently, from (49)-(52) and by regrouping terms and taking cancellations into account we obtain

$$
\begin{aligned}
& U_{N, \gamma, n}(t, s)-\exp \left[-i h H_{0, N}\right] \exp \left[-i h V_{N}(s+\gamma h)\right] \\
& =i \int_{s+\gamma h}^{s+(\gamma+1) h} d \tau_{1}\left(V_{N}(s+\gamma h)-V_{N}\left(\tau_{1}\right)\right)+O_{N}\left(h^{2}\right)
\end{aligned}
$$


as $h \rightarrow 0_{+}$, so that in order to prove the first part of (47) it is sufficient to have

$$
\int_{\gamma h}^{(\gamma+1) h} d \tau\left(V_{N}(s+\gamma h)-V_{N}(s+\tau)\right)=O_{N}\left(h^{2}\right)
$$

where we performed an elementary change of variables in the integral. For the matrix elements of the integrand in (54) we now have

$$
\begin{gathered}
V_{N, m, m^{\prime}}(s+\gamma h)-V_{N, m, m^{\prime}}(s+\tau) \\
=\lambda v_{m, m^{\prime}}(a(s+\gamma h)-a(s+\tau))
\end{gathered}
$$

for all $m, m^{\prime} \in\{0, \ldots, N-1\}$ and for some $v_{m, m^{\prime}} \in \mathbb{R}$ according to (38). Furthermore we have

$$
\int_{\gamma h}^{(\gamma+1) h} d \tau(\tau-\gamma h)=\frac{h^{2}}{2} .
$$

Consequently from (55), the Lipschitz continuity of $a,(56)$ and the equivalence of all matrix norms on $E_{N}(\mathbb{R}, \mathbb{C})$ we obtain the estimates

$$
\begin{aligned}
& \int_{\gamma h}^{(\gamma+1) h} d \tau\left\|V_{N}(s+\gamma h)-V_{N}(s+\tau)\right\| \\
& \leq O_{N}(1) \sum_{m, m^{\prime}=0}^{N-1} \int_{\gamma h}^{(\gamma+1) h} d \tau\left|V_{N, m, m^{\prime}}(s+\gamma h)-V_{N, m, m^{\prime}}(s+\tau)\right| \\
& \leq O_{N}(1) \sum_{m, m^{\prime}=0}^{N-1}\left|v_{m, m^{\prime}}\right| \int_{\gamma h}^{(\gamma+1) h} d \tau(\tau-\gamma h)=O_{N}(1) h^{2}
\end{aligned}
$$

as $h \rightarrow 0_{+}$uniformly in $\gamma$, which is (54) so that the first part of (47) indeed holds.

Replacing now $V_{N}(s+\gamma h)$ by $W_{N, \gamma}(s, h)$ in (53) gives

$$
\begin{aligned}
& U_{N, \gamma, n}(t, s)-\exp \left[-i h H_{0, N}\right] \exp \left[-i h W_{N, \gamma}(s, h)\right] \\
& =i \int_{s+\gamma h}^{s+(\gamma+1) h} d \tau_{1}\left(W_{N, \gamma}(s, h)-V_{N}\left(\tau_{1}\right)\right)+O_{N}\left(h^{2}\right)
\end{aligned}
$$

where this time

$$
\int_{\gamma h}^{(\gamma+1) h} d \tau\left(W_{N, \gamma}(s, h)-V_{N}(s+\tau)\right)=O_{N}\left(h^{3}\right) .
$$

This last relation is indeed a consequence of (29) and a standard trapezoidal rule estimate of the form

$$
\int_{\gamma h}^{(\gamma+1) h} d \tau a(s+\tau)=\frac{h}{2}\left(a(s+\gamma h)+a(s+(\gamma+1) h)+O\left(h^{3}\right)\right.
$$


for the amplitude function $a$, since we have

$$
\begin{aligned}
& \left\|\int_{\gamma h}^{(\gamma+1) h} d \tau\left(W_{N, \gamma}(s, h)-V_{N}(s+\tau)\right)\right\| \\
& \leq O_{N}(1) \sum_{m, m^{\prime}=0}^{N-1}\left|\int_{\gamma h}^{(\gamma+1) h} d \tau\left(W_{N, \gamma, m, m^{\prime}}(s, h)-V_{N, m, m^{\prime}}(s+\tau)\right)\right| \\
& =O_{N}(1) \sum_{m, m^{\prime}=0}^{N-1}\left|v_{m, m^{\prime}}\right| \mid \frac{h}{2}\left(a(s+\gamma h)+a(s+(\gamma+1) h)-\int_{\gamma h}^{(\gamma+1) h} d \tau a(s+\tau) \mid\right. \\
& =O_{N}\left(h^{3}\right) .
\end{aligned}
$$

But in spite of this estimate the second term on the second line of (57) remains $O_{N}\left(h^{2}\right)$, so that the second equality in (47) also holds without any room for improvement.

The situation is quite different regarding the proof of (48). In this case we start with

$$
\begin{aligned}
& U_{N, \gamma, n}(t, s) \\
& =\mathbb{I}_{N}-i \int_{s+\gamma h}^{s+(\gamma+1) h} d \tau\left(H_{0, N}+V_{N}\left(\tau_{1}\right)\right) \\
& -\int_{s+\gamma h}^{s+(\gamma+1) h} d \tau_{1} \int_{s+\gamma h}^{\tau_{1}} d \tau_{2}\left(H_{0, N}+V_{N}\left(\tau_{1}\right)\right)\left(H_{0, N}+V_{N}\left(\tau_{2}\right)\right)+O_{N}\left(h^{3}\right) .
\end{aligned}
$$

Furthermore we have

$$
\begin{aligned}
& \exp \left[-i h H_{0, N}\right] \exp \left[-i h W_{N, \gamma}(s, h)\right] \exp \left[\frac{h^{2}}{2}\left[H_{0, N}, W_{N, \gamma}(s, h)\right]_{-}\right] \\
& =\mathbb{I}_{N}-i h\left(H_{0, N}+W_{N, \gamma}(s, h)\right)-\frac{h^{2}}{2}\left(H_{0, N}+W_{N, \gamma}(s, h)\right)^{2}+O_{N}\left(h^{3}\right)
\end{aligned}
$$

again by expanding exponentials. Therefore, by lumping terms together and by making appropriate changes of variables in the integrals we obtain

$$
\begin{aligned}
& U_{N, \gamma, n}(t, s)-\exp \left[-i h H_{0, N}\right] \exp \left[-i h W_{N, \gamma}(s, h)\right] \exp \left[\frac{h^{2}}{2}\left[H_{0, N}, W_{N, \gamma}(s, h)\right]_{-}\right] \\
& =i \int_{\gamma h}^{(\gamma+1) h} d \tau\left(W_{N, \gamma}(s, h)-V_{N}(s+\tau)\right) \\
& -\int_{\gamma h}^{(\gamma+1) h} d \tau \int_{\gamma h}^{\tau} d \sigma\left(\left(H_{0, N}+V_{N}(s+\tau)\right)\left(H_{0, N}+V_{N}(s+\sigma)\right)-\left(H_{0, N}+W_{N, \gamma}(s, h)\right)^{2}\right) \\
& +O_{N}\left(h^{3}\right) .
\end{aligned}
$$


We proceed by decomposing this last integrand as

$$
\begin{aligned}
& \left(H_{0, N}+V_{N}(s+\tau)\right)\left(H_{0, N}+V_{N}(s+\sigma)\right)-\left(H_{0, N}+W_{N, \gamma}(s, h)\right)^{2} \\
& =\left(H_{0, N}+V_{N}(s+\tau)\right)\left(V_{N}(s+\sigma)-W_{N, \gamma}(s, h)\right) \\
& +\left(V_{N}(s+\tau)-W_{N, \gamma}(s, h)\right)\left(H_{0, N}+W_{N, \gamma}(s, h)\right)
\end{aligned}
$$

and substitute this expression in the above integral; in this way we get

$$
\begin{aligned}
& U_{N, \gamma, n}(t, s)-\exp \left[-i h H_{0, N}\right] \exp \left[-i h W_{N, \gamma}(s, h)\right] \exp \left[\frac{h^{2}}{2}\left[H_{0, N}, W_{N, \gamma}(s, h)\right]_{-}\right] \\
& =i \int_{\gamma h}^{(\gamma+1) h} d \tau\left(W_{N, \gamma}(s, h)-V_{N}(s+\tau)\right) \\
& +\int_{\gamma h}^{(\gamma+1) h} d \tau\left(H_{0, N}+V_{N}(s+\tau)\right) \int_{\gamma h}^{\tau} d \sigma\left(W_{N, \gamma}(s, h)-V_{N}(s+\sigma)\right) \\
& +\int_{\gamma h}^{(\gamma+1) h} d \tau(\tau-\gamma h)\left(W_{N, \gamma}(s, h)-V_{N}(s+\tau)\right)\left(H_{0, N}+W_{N, \gamma}(s, h)\right)+O_{N}\left(h^{3}\right) .
\end{aligned}
$$

We already know that (58) holds, so that it remains to estimate the second and third terms on the right-hand side of the preceding expression. Regarding the second term we notice that

$$
\int_{\gamma h}^{\tau} d \sigma\left(W_{N, \gamma}(s, h)-V_{N}(s+\sigma)\right)=O_{N}\left((\tau-\gamma h)^{3}\right)
$$

which can be obtained exactly as we proved (58), so that (50) and (60) lead to

$$
\begin{aligned}
& \int_{\gamma h}^{(\gamma+1) h} d \tau\left\|\left(H_{0, N}+V_{N}(s+\tau)\right) \int_{\gamma h}^{\tau} d \sigma\left(W_{N, \gamma}(s, h)-V_{N}(s+\sigma)\right)\right\| \\
& \leq O_{N}(1) \int_{\gamma h}^{(\gamma+1) h} d \tau\left\|\int_{\gamma h}^{\tau} d \sigma\left(W_{N, \gamma}(s, h)-V_{N}(s+\sigma)\right)\right\| \\
& \leq O_{N}(1) \int_{\gamma h}^{(\gamma+1) h} d \tau(\tau-\gamma h)^{3}=O_{N}\left(h^{4}\right)
\end{aligned}
$$

uniformly in $\gamma$, which is more than we need. Finally, for the third term we get

$$
\int_{\gamma h}^{(\gamma+1) h} d \tau(\tau-\gamma h)\left(W_{N, \gamma}(s, h)-V_{N}(s+\tau)\right)\left(H_{0, N}+W_{N, \gamma}(s, h)\right)=O_{N}\left(h^{3}\right)
$$

uniformly in $\gamma$ from entirely similar arguments based this time on the trapezoidal rule estimate

$$
\int_{\gamma h}^{(\gamma+1) h} d \tau(\tau-\gamma h) a(s+\tau)=\frac{h^{2}}{2} a(s+(\gamma+1) h)+O\left(h^{3}\right)
$$


and the Lipschitz continuity of $a$.

The proof of the second part of (48) is identical and thereby omitted.

We are now ready for the following:

Proof of the Theorem. Let us begin by proving (41). According to (46) we have

$$
\begin{aligned}
& U_{N}(t, s)-\prod_{\gamma=n-1}^{0} \exp \left[-i h H_{0, N}\right] \exp \left[-i h V_{N}(s+\gamma h)\right] \\
& =\prod_{\gamma=n-1}^{0} U_{N, \gamma, n}(t, s)-\prod_{\gamma=n-1}^{0} \exp \left[-i h H_{0, N}\right] \exp \left[-i h V_{N}(s+\gamma h)\right]
\end{aligned}
$$

Furthermore, for each $\gamma \in\{0, \ldots, n-1\}$ let us introduce momentarily the shorthand notation

$$
A_{\gamma}:=U_{N, \gamma, n}(t, s)
$$

and

$$
B_{\gamma}:=\exp \left[-i h H_{0, N}\right] \exp \left[-i h V_{N}(s+\gamma h)\right] .
$$

For every $n \geq 3$ we then have the decomposition formula

$$
\begin{aligned}
& \prod_{\gamma=n-1}^{0} A_{\gamma}-\prod_{\gamma=n-1}^{0} B_{\gamma} \\
= & \prod_{\beta=n-1}^{1} B_{\beta} \times\left(A_{0}-B_{0}\right) \\
+ & \sum_{\gamma=1}^{n-2} \prod_{\beta=n-1}^{\gamma+1} B_{\beta} \times\left(A_{\gamma}-B_{\gamma}\right) \times \prod_{\alpha=\gamma-1}^{0} A_{\alpha} \\
+ & \left(A_{n-1}-B_{n-1}\right) \times \prod_{\alpha=n-2}^{0} A_{\alpha}
\end{aligned}
$$

which can easily be checked directly, and therefore

$$
\left\|\prod_{\gamma=n-1}^{0} A_{\gamma}-\prod_{\gamma=n-1}^{0} B_{\gamma}\right\| \leq \sum_{\gamma=0}^{n-1}\left\|A_{\gamma}-B_{\gamma}\right\|
$$

since the operators $A_{\gamma}$ and $B_{\gamma}$ are all unitary with respect to the supremumnorm. Consequently from (62), (47) and remembering the definition of $h$ we 
obtain

$$
\begin{aligned}
& \left\|U_{N}(t, s)-\prod_{\gamma=n-1}^{0} \exp \left[-i h H_{0, N}\right] \exp \left[-i h V_{N}(s+\gamma h)\right]\right\| \\
& \leq \sum_{\gamma=0}^{n-1}\left\|U_{N, \gamma, n}(t, s)-\exp \left[-i h H_{0, N}\right] \exp \left[-i h V_{N}(s+\gamma h)\right]\right\| \\
& =n O_{N}\left(h^{2}\right)=O_{N}(h)
\end{aligned}
$$

since the estimates of Lemma 3 are all uniform in $\gamma$, which is the desired result. The proofs of the remaining equalities in (42) are entirely similar and thereby omitted.

Remarks. (1) We can prove that

$$
\begin{aligned}
& U_{N}(t, s) \\
& =\prod_{\gamma=n-1}^{0} \exp \left[-i h\left(H_{0, N}+V_{N}(s+\gamma h)\right)\right]+O_{N}(h) \\
& =\prod_{\gamma=n-1}^{0} \exp \left[-i h\left(H_{0, N}+W_{N, \gamma}(s, h)\right)\right]+O_{N}\left(h^{2}\right)
\end{aligned}
$$

when $h \rightarrow 0_{+}$in exactly the same way as we proved the above theorem. However, whereas (65) shows how to reconstruct the true dynamics from the basic exponentials, it has little value from a computational point of view.

(2) The second part of the proof of Lemma 3 shows why we would have obtained the slower convergence rates (44) had we kept $V_{N}(s+\gamma h)$ instead of $W_{N, \gamma}(s, h)$ in (59): whereas the second and third terms on the right-hand side of that expression would still have been $O_{N}\left(h^{3}\right)$, the very first term would have remained $O_{N}\left(h^{2}\right)$ according to (54).

(3) The fact that all the approximating operators are unitary with respect to the supremum-norm is of course an essential ingredient of the above proof. If those operators were not isometries, the $n$-dependence in (63) could not be controlled and (64) not be established. It turns out that such a norm-preserving property will also be crucial for numerical stability purposes, as we will see in Section 3.

It is now easy to convert the statements of the theorem into estimates for the solutions to (23). Let us write $\|\cdot\|_{2}$ for the norm in $L^{2}(\mathbb{R}, \mathbb{C})$; since $\left\|v_{N}\right\|_{2} \leq\|v\|_{2}$ by the very definition of $v_{N}$, the above theorem then immediately implies the following result where $u_{N, n}^{T K}, u_{N, n}^{B C H}$ and $u_{N, n}^{Y}$ are given by (26), (27) and (28), respectively, this time all three with $s=0$ :

Corollary. For every $t \in[0, T]$ we have the estimates

$$
\left\|u_{N}(t)-u_{N, n}^{T K}(t)\right\|_{2} \leq \frac{\|v\|_{2}}{n}
$$




$$
\left\|u_{N}(t)-u_{N, n}^{B C H}(t)\right\|_{2} \leq \frac{\|v\|_{2}}{n^{2}}
$$

and

$$
\left\|u_{N}(t)-u_{N, n}^{Y}(t)\right\|_{2} \leq \frac{\|v\|_{2}}{n^{2}}
$$

as $n \rightarrow+\infty$, uniformly in $N \in \mathbb{N}^{+}$.

Remark. According to the last part of the third remark following the main theorem, we also have

$$
\left\|u_{N}(t)-u_{N, n}^{Y^{\prime}}(t)\right\|_{2} \leq \frac{\|v\|_{2}}{n^{2}}
$$

as $n \rightarrow+\infty$ uniformly in $N \in \mathbb{N}^{+}$, where

$u_{N, n}^{Y^{\prime}}(t):=\prod_{\gamma=n-1}^{0} \exp \left[-i \frac{h}{2} W_{N, \gamma}(0, h)\right] \exp \left[-i h H_{0, N}\right] \exp \left[-i \frac{h}{2} W_{N, \gamma}(0, h)\right] v_{N}$.

The proof is identical to that of the above corollary.

In the next section we compute the solutions to Problems (2) and (30) according to the outline of Section 1, and then compare those results with the analytical statements of this section.

\section{Some Numerical Results}

The computation of the solution to

$$
\begin{aligned}
i \frac{\partial u(x, t)}{\partial t} & =H u(x, t), \quad(x, t) \in \mathbb{R} \times(0, T], \\
u(x, 0) & =v(x), \quad x \in \mathbb{R}
\end{aligned}
$$

where

$$
H:=-\frac{1}{2} \frac{\partial^{2}}{\partial x^{2}}+\frac{1}{2} x^{2}+\lambda x^{4}
$$

and where $v$ satisfies

$$
H v(x)=E_{0} v(x)
$$

with $E_{0}$ the smallest eigenvalue in (73), first calls for the computation and for very good analytical approximations of $E_{0}$ and $v$ whether we carry out the computation by means of (32) or (33)-(35), since (73) is not exactly solvable when $\lambda>0$. While we carry out the computation of $E_{0}$ and $v$ by means of MatLab's eigs, our choice for the corresponding approximations rests on the observation that (73) reduces to (36) when $\lambda=0$, whose exact solution for $m=0$ is $E_{0}=\frac{1}{2}$ with

$$
\mathrm{h}_{0}(x)=\pi^{-\frac{1}{4}} e^{-\frac{x^{2}}{2}} .
$$


It is then natural to embed (74) into the one-parameter family of Gaussian curves given by

$$
\mathrm{h}_{0, \alpha}(x):=\left(\frac{\alpha}{\pi}\right)^{\frac{1}{4}} e^{-\frac{\alpha x^{2}}{2}}
$$

where $\alpha>0$, and to choose the function

$$
v_{\alpha^{*}}(x):=\mathrm{h}_{0, \alpha^{*}}(x)
$$

to approximate $v$ where $\alpha^{*}$ is the solution to the minimization problem

$$
E_{0}^{*}:=\min _{\alpha>0}\left(H \mathrm{~h}_{0, \alpha}, \mathrm{h}_{0, \alpha}\right)_{2} .
$$

Indeed for $\lambda=0$ this procedure leads to the exact value of the smallest eigenvalue corresponding to $\alpha^{*}=1$, while in the general case we have the following result:

Lemma 4. For all $\alpha>0$ and $\lambda \geq 0$ we have

$$
E_{0, \alpha}:=\left(H \mathrm{~h}_{0, \alpha}, \mathrm{h}_{0, \alpha}\right)_{2}=\frac{1}{4}\left(\frac{3 \lambda}{\alpha^{2}}+\frac{1}{\alpha}+\alpha\right),
$$

and furthermore the following statements hold:

(a) For every $\lambda \in\left[0, \frac{\sqrt{3}}{27}\right]$ we have

$$
E_{0}^{*}=E_{0, \alpha^{*}}
$$

with

$$
\alpha^{*}=\frac{2 \sqrt{3}}{3} \cos \frac{\varphi}{3}
$$

and

$$
\varphi=\arccos 9 \sqrt{3} \lambda .
$$

In particular, for $\lambda=\frac{\sqrt{3}}{27}$ this gives $\alpha^{*}=\frac{2 \sqrt{3}}{3}$.

(b) For every $\lambda \in\left(\frac{\sqrt{3}}{27},+\infty\right)$ we have

$$
E_{0}^{*}=E_{0, \alpha^{*}}
$$

where

$$
\alpha^{*}=\left(3 \lambda+\left(9 \lambda^{2}-\frac{1}{27}\right)^{\frac{1}{2}}\right)^{\frac{1}{3}}+\left(3 \lambda-\left(9 \lambda^{2}-\frac{1}{27}\right)^{\frac{1}{2}}\right)^{\frac{1}{3}}
$$

Proof. From (72) and (75) we obtain

$$
H \mathrm{~h}_{0, \alpha}(x)=\frac{1}{2}\left(\alpha-\left(\alpha^{2}-1\right) x^{2}+2 \lambda x^{4}\right) \mathrm{h}_{0, \alpha}(x),
$$

so that (76) follows from the definition of $E_{0, \alpha}$ through the values of the second and fourth moments of a Gaussian distribution. Furthermore, from (76) it is 
plain that the function $\alpha \mapsto E_{0, \alpha}$ is convex for every $\lambda \geq 0$, and that $\alpha^{*}>0$ is a zero of its first derivative if, and only if,

$$
\alpha^{* 3}-\alpha^{*}-6 \lambda=0 .
$$

But if $\lambda \in\left[0, \frac{\sqrt{3}}{27}\right]$ the only positive root of this equation is (78), while if $\lambda \in\left(\frac{\sqrt{3}}{27},+\infty\right)$ its only positive root is $(80)$.

In what follows we refer to $v_{\alpha^{*}}$ as the best Gaussian approximation for $v$, thus having

$$
v_{\alpha^{*}}(x)=\left(\frac{\alpha^{*}}{\pi}\right)^{\frac{1}{4}} e^{-\frac{\alpha^{*} x^{2}}{2}}
$$

where $\alpha^{*} \geq 1$ is given by (78) or (80) depending on the value of $\lambda$, with (77) and (79) as approximations for the corresponding eigenvalues.

Aside from providing explicit expressions, relations (77), (79) and (81) turn out to be very close to the actual solution to (73) indeed. In order to see this we first show in Figure 1 the size of the expansion coefficients of the eigenfunction $v$ in (73) relative to the Hermite basis $\left(\mathrm{h}_{m}\right)_{m \in \mathbb{N}}$ which we computed by means of MatLab's eigs, and in Figure 2 the corresponding matrix norms $\left\|H_{0, N}\right\|$, $\left\|V_{N}\right\|,\left\|H_{N}\right\|,\left\|C_{N}\right\|$ and some condition numbers as a function of $N$, where $C_{N}:=\left[H_{0, N}, V_{N}\right]_{-}:$

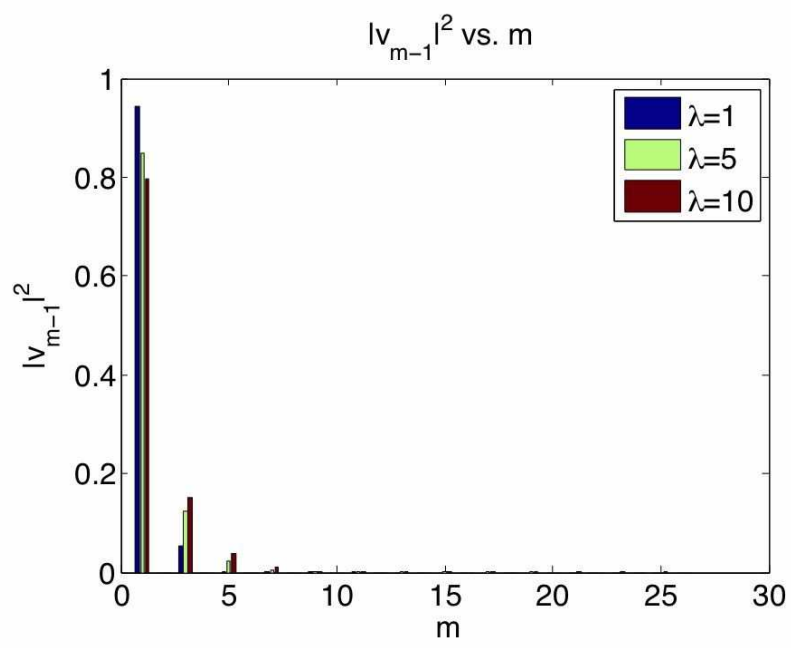

Figure 1: size of the expansion coefficients of $v$ with respect to the Hermite basis $\left(\mathrm{h}_{m}\right)_{m \in \mathbb{N}}$ for $\lambda=1,5,10$. The rapid fall-off shows that for $m \geq N \simeq 25$ we may assume $v_{m}=0$, for then $\sum_{m \geq N}\left|v_{m}\right|^{2} \lesssim 10^{-5}$. 


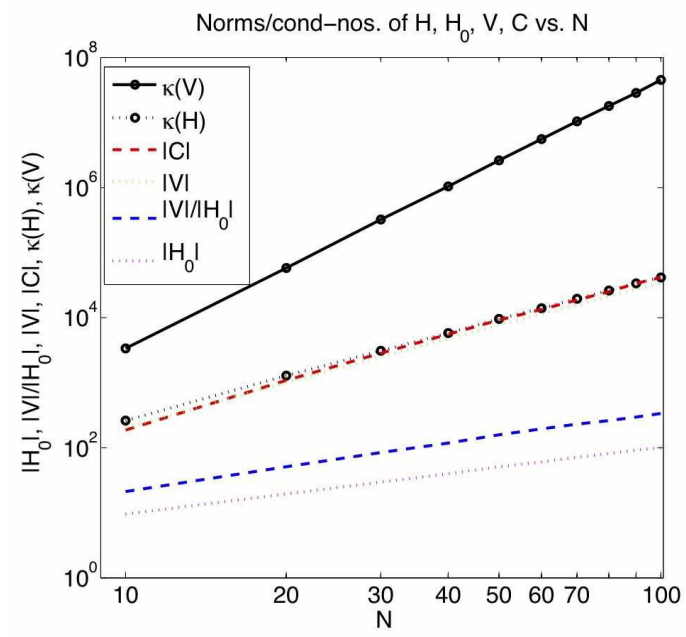

Figure 2: various norms and condition numbers as a function of $N$. The norm $\left\|H_{0, N}\right\|$ grows linearly, while $\left\|V_{N}\right\|$ and $\left\|H_{N}\right\|$ grow roughly as $N^{2.2}$ and $\left\|C_{N}\right\|$ as $N^{2.3}$. The condition number $\varkappa\left(C_{N}\right)$ is not shown since $C_{N}$ is singular for about seventy-five percent of the values from $N=10$ to $N=100$.

Indeed the latter diagram is related to the very important issue of knowing how large we can choose $N$ in the Faedo-Galerkin scheme for our results to be accurate when using MatLab's eigs, that is, of having

$$
v_{N}=\sum_{m=0}^{N-1} v_{m} \mathrm{~h}_{m}
$$

to accurately represent $v$. Furthermore, it also turns out that the rapid fall-off of $v_{m}$ as illustrated in Figure 1 can be well duplicated analytically by means of the best Gaussian approximation $v_{\alpha^{*}}$. Indeed we remark that since $v_{\alpha^{*}} \in L^{2}(\mathbb{R}, \mathbb{C})$ is even, and since the Hermite functions (22) have the parity of $m$, we may decompose (81) as

$$
v_{\alpha^{*}}=v_{\alpha^{*}, N}+\sum_{m=N}^{+\infty} v_{2 m, \alpha^{*}} \mathrm{~h}_{2 m}
$$

where

$$
v_{\alpha^{*}, N}:=\sum_{m=0}^{N-1} v_{2 m, \alpha^{*}} \mathrm{~h}_{2 m}
$$

and

$$
\lim _{N \rightarrow+\infty} \sum_{m=N}^{+\infty}\left|v_{2 m, \alpha^{*}}\right|^{2}=0 .
$$

Moreover, the advantage of having $v_{\alpha^{*}}$ as a Gaussian curve makes it possible to compute the coefficients $v_{2 m, \alpha^{*}}$ explicitly and thereby obtain the following rapid decay estimate for the series (84): 
Lemma 5. For $\alpha^{*} \geq 1$ as in the preceding lemma we have

$$
\sum_{m=N}^{+\infty}\left|v_{2 m, \alpha^{*}}\right|^{2} \leq \frac{\alpha^{*}+1}{\sqrt{2 \pi N \alpha^{*}}}\left(\frac{\alpha^{*}-1}{\alpha^{*}+1}\right)^{N}
$$

for every $N \in \mathbb{N}^{+}$.

Proof. From Relation 7.373-2 in Chapter 7 of [15] and an elementary change of variables we get

$$
\begin{aligned}
& \int_{\mathbb{R}} d x e^{-\frac{\alpha^{*}+1}{2} x^{2}} \mathrm{H}_{2 m}(x) \\
= & \sqrt{2 \pi} \frac{(2 m) !\left(1-\alpha^{*}\right)^{m}}{m !\left(1+\alpha^{*}\right)^{m+\frac{1}{2}}}
\end{aligned}
$$

for every $m \in \mathbb{N}$, so that (22) and (81) lead to

$$
\begin{aligned}
v_{2 m, \alpha^{*}} & =\int_{\mathbb{R}} d x v_{\alpha^{*}}(x) \mathrm{h}_{2 m}(x) \\
& =2^{\frac{1-2 m}{2}} \frac{\sqrt{(2 m) ! \sqrt[4]{\alpha^{*}}\left(1-\alpha^{*}\right)^{m}}}{m !\left(1+\alpha^{*}\right)^{m+\frac{1}{2}}},
\end{aligned}
$$

which gives

$$
\left|v_{2 m, \alpha^{*}}\right|^{2}=\frac{(2 m) !}{2^{2 m}(m !)^{2}} \times \frac{2 \sqrt{\alpha^{*}}\left(\alpha^{*}-1\right)^{2 m}}{\left(\alpha^{*}+1\right)^{2 m+1}} .
$$

Next, we prove that the inequality

$$
\frac{(2 m) !}{2^{2 m}(m !)^{2}} \leq \frac{1}{\sqrt{\pi m}}
$$

holds for every $m \in \mathbb{N}^{+}$. To this end we recall that

$$
m !=\left(\frac{m}{e}\right)^{m} \sqrt{2 \pi m} \exp \left[-\int_{m}^{+\infty} d x \frac{p(x)}{x^{2}}\right]
$$

as a consequence of Euler's summation formula, where $p:[0,+\infty) \mapsto \mathbb{R}^{-}$is periodic with period one and

$$
p(x)=\frac{x(x-1)}{2}
$$

for every $x \in[0,1]$ (see, for instance, Theorem 15.18 in Chapter 15 of [2]). By applying (88) to both factorials on the left-hand side of (87) we obtain

$$
\frac{(2 m) !}{2^{2 m}(m !)^{2}}=\frac{1}{\sqrt{\pi m}} \exp \left[\int_{m}^{+\infty} d x \frac{p(x)}{x^{2}}+\int_{m}^{2 m} d x \frac{p(x)}{x^{2}}\right],
$$


which implies (87) since $p(x) \leq 0$ for every $x$. We now substitute (87) into (86) and take $m \geq N$ into account to get

$$
\begin{aligned}
& \sum_{m=N}^{+\infty}\left|v_{2 m, \alpha^{*}}\right|^{2} \\
\leq & \frac{2 \sqrt{\alpha^{*}}}{\left(\alpha^{*}+1\right) \sqrt{\pi N}} \sum_{m=N}^{+\infty}\left(\frac{\alpha^{*}-1}{\alpha^{*}+1}\right)^{2 m} \\
= & \frac{\alpha^{*}+1}{\sqrt{4 \pi N \alpha^{*}}}\left(\frac{\alpha^{*}-1}{\alpha^{*}+1}\right)^{2 N} \\
\leq & \frac{\alpha^{*}+1}{\sqrt{2 \pi N \alpha^{*}}}\left(\frac{\alpha^{*}-1}{\alpha^{*}+1}\right)^{N}
\end{aligned}
$$

after summing explicitly the tail of the geometric series.

The preceding result is very useful in that it allows us to estimate $N$ as a function of the degree of precision we want for (82) to approximate $v$. Thus, if we impose for instance that

$$
\frac{\alpha^{*}+1}{\sqrt{2 \pi N \alpha^{*}}}\left(\frac{\alpha^{*}-1}{\alpha^{*}+1}\right)^{N} \lesssim 10^{-5}
$$

then we obtain $N \simeq 23$, a result of the same order of magnitude as that illustrated in Figure 1. Moreover, Figure 3 below shows that the value $E_{0}^{*}$ is very closely related to the computed eigenvalue $E_{0}$ in (73), and that the two curves are essentially indistinguishable for small values of $\lambda$ :

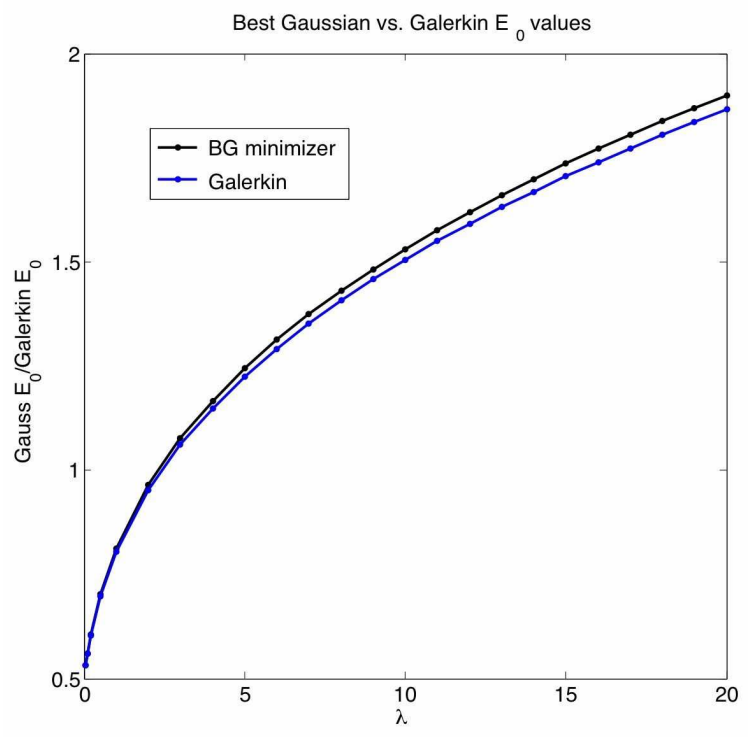

Figure 3: the Gaussian value $E_{0}^{*}$ compared with the computed eigenvalue $E_{0}$ in (73), as a function of the anharmonic coupling. 
Our preliminary step is thereby completed, that is, we have obtained computed values for $E_{0}$ and $v$ in (73) as well as very good analytical approximations for them. We will get other estimates for $E_{0}$ and $v$ by means of a formal WKB approximation in the Appendix.

Remark. Actually, within our Faedo-Galerkin scheme MatLab's eigs allows us to compute all the nontrivial solutions to the differential equation

$$
\left(-\frac{1}{2} \frac{d^{2}}{d x^{2}}+\frac{1}{2} x^{2}+\lambda x^{4}\right) v(x)=E v(x)
$$

having their $L^{2}\left(\mathbb{R}, \mathbb{C}\right.$ )-norm equal to one, that is, all the eigenvalues $E_{m}$ and the corresponding normalized eigenfunctions of the differential operator in (89), in addition to $E_{0}$ and $v$. In Figure 4 below we display parts of the anharmonic spectra as a function of $m$ for several values of $\lambda$, while in Figure 5 we plot five of those eigenvalues as a function of $\lambda$. In Figure 6 we show how large we have to choose $N:=N_{\text {stop }}$ in our Faedo-Galerkin scheme in order to accurately get the $m^{t h}$ eigenvalue and the $m^{t h}$ eigenfunction within the precision desired when $\lambda=1$. It is interesting to note that from all the computations carried out thus far, the dimension $N_{\text {stop }}$ of the Galerkin subspace has to be increased when the anharmonic coupling $\lambda$ becomes large, and in that case our results are more sensitive to the chosen step size as well.

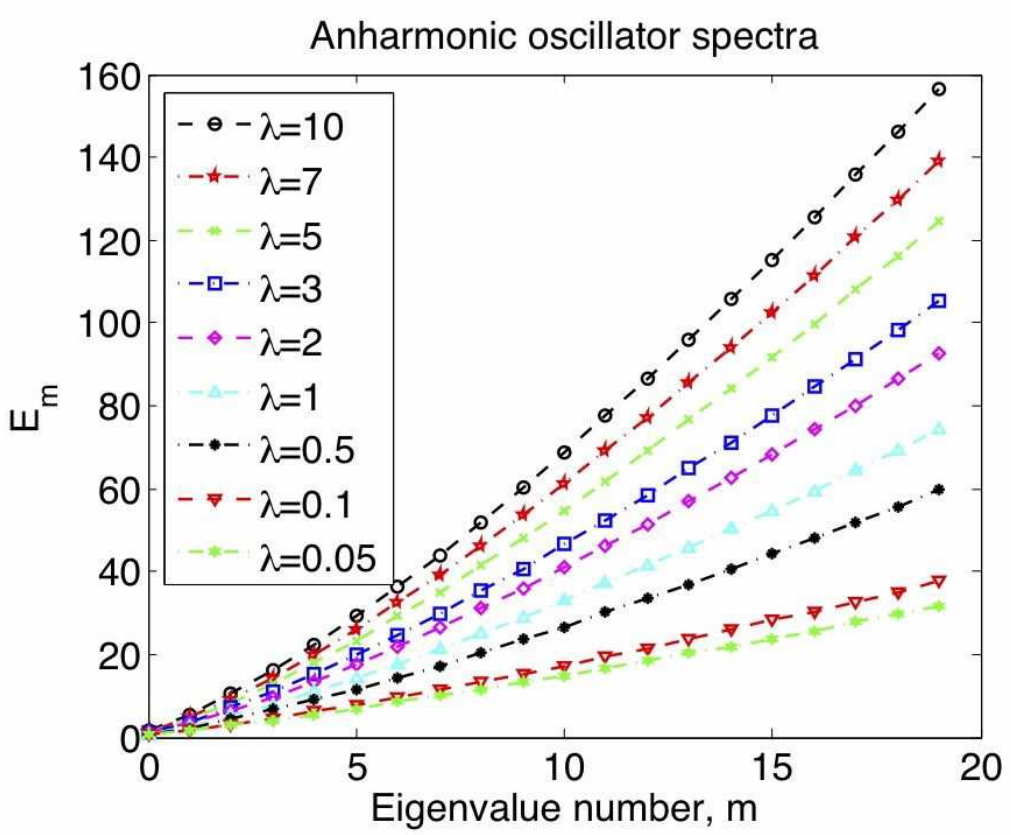

Figure 4: the first twenty eigenvalues $E_{m}$ of the differential operator in (89) for various values of $\lambda$. Away from $\lambda=0, E_{m}$ is no longer linear in $m$. 


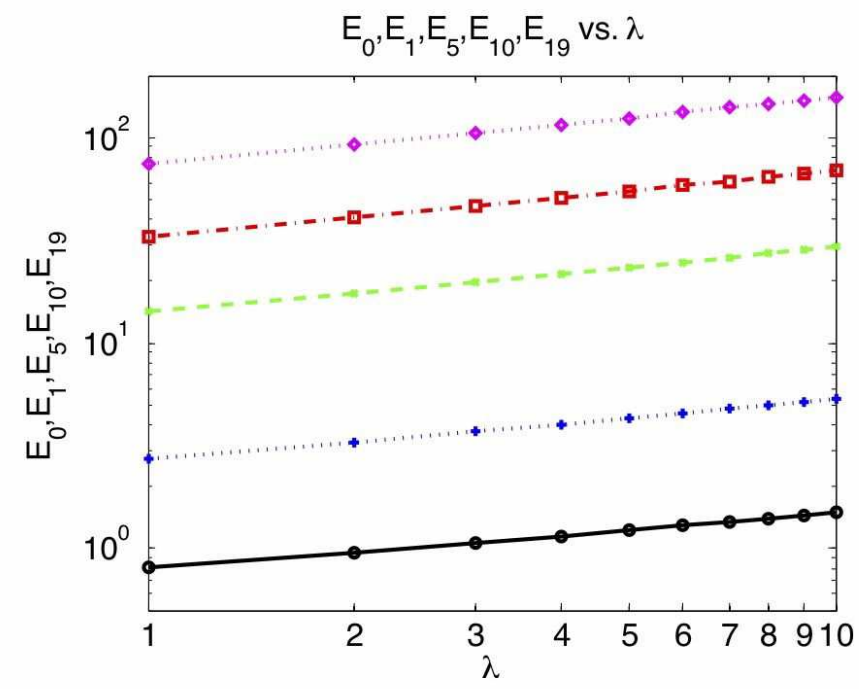

Figure 5: five eigenvalues of (89) as a function of the anharmonic coupling.

Detailed fits using polyfit show $E_{m} \simeq c_{m} \lambda^{\nu}$ where $0.31 \leq \nu \leq 0.325$.

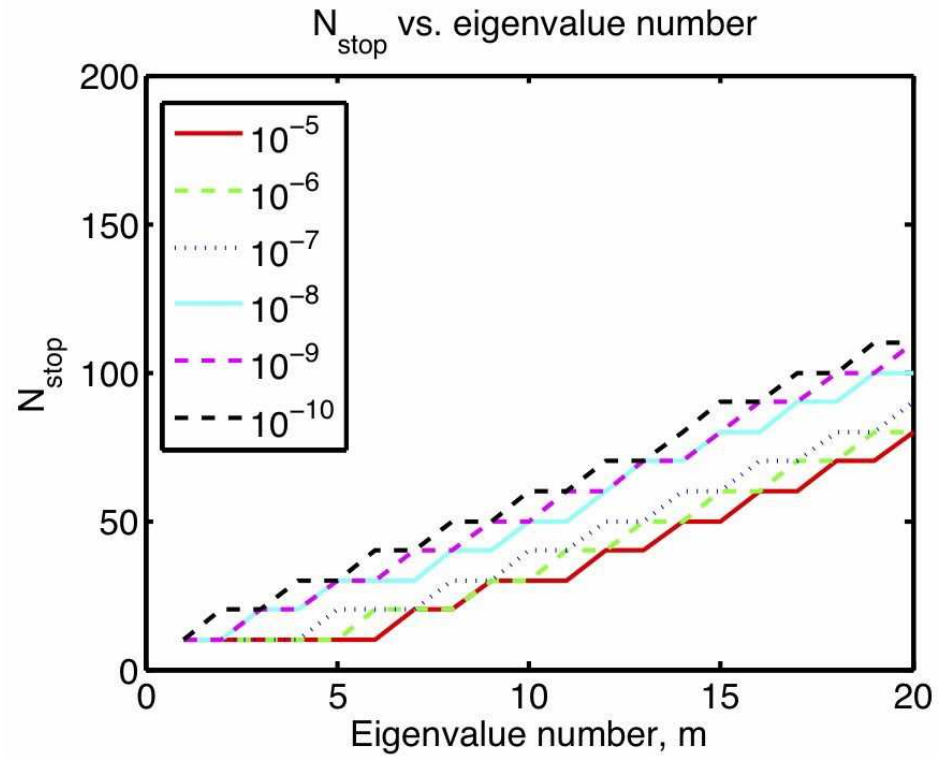

Figure 6: the number $N_{\text {stop }}$ of the Faedo-Galerkin scheme needed to accurately capture the $m^{t h}$ eigenvalue and the $m^{t h}$ eigenfunction in (89) for $\lambda=1$, versus the precision desired: the larger $m$ is, the larger $N_{\text {stop }}$ ought to be.

Next, we describe the results of our actual computation of the solution to (71)(73) by means of (32) and (33)-(35), in which we choose the initial condition 
to be the solution to (73) computed with MatLab's eigs. In all the forthcoming figures of this section the results obtained from the Yoshida splitting are indifferently labeled YOS or YOS2, and all the exponentials in (32) and (33)-(35) are computed by means of the routine MatLab's expm which uses

$$
\operatorname{expm}(A)=\left(P^{-1}(-A / m) P(A / m)\right)^{m}
$$

through the method called scaling and squaring where $P$ is a thirteenth degree real polynomial (see [18]). By repeated squaring of the inner argument the required power $m$ can be computed in $q$ steps when $m=2^{q}$. It turns out that this method is surprisingly very robust, while we are acutely aware of the longstanding difficulties of computing matrix exponentials (see, for instance, [21]); when applied to the difference between (32) and (33) on the one hand, and between (32) and (35) on the other hand, that method leads to the following figure which shows the corresponding root mean square errors as a function of the uniform step size $h$ defined in Section 1 , for $\lambda=1, N=50$ and $t=20$; we write $u_{\mathrm{ex}}$ for the computed solution (32):

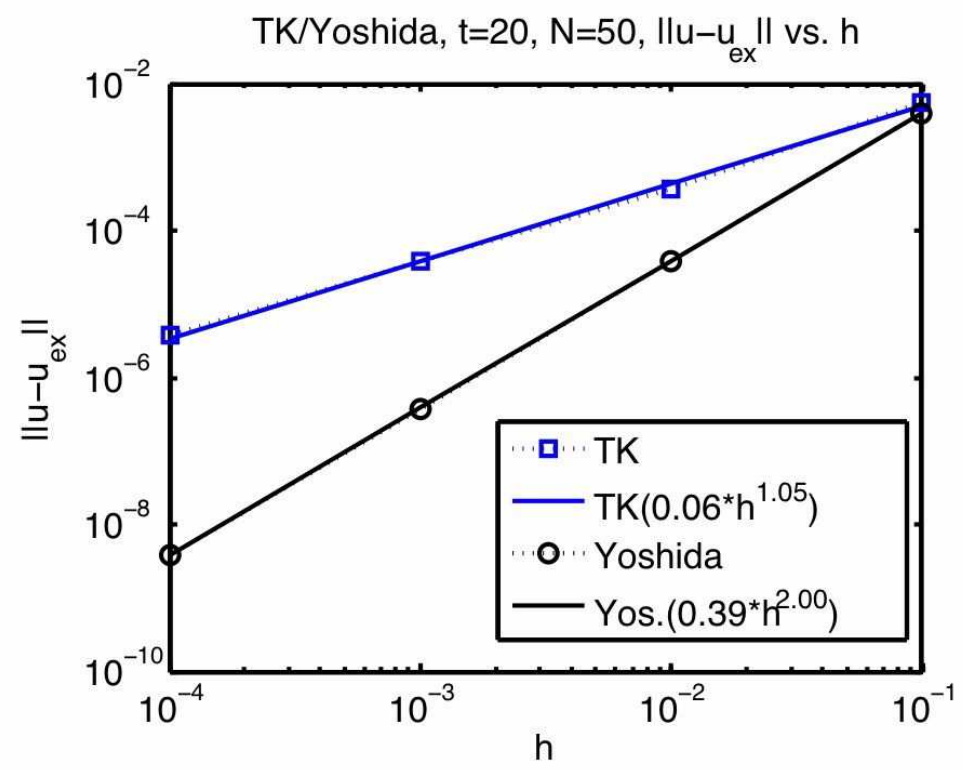

Figure 7: comparison of the RMS errors of the TK-splitting and the Y-splitting with (32) for the autonomous problem, for $N=50, \lambda=1$ and $t=20$. Straight lines are two-parameter fits.

REMARKs. (1) In Figure 7 we do not display the computation of the root mean square error between (32) and (34) for, within the accuracy of the printed numerical data and that of the plotter, that computation leads exactly to the same curve as that relative to the $Y$-splitting. The same observation holds for 
the root mean square error between (32) and the autonomous version of (70), that is,

$$
u_{N, n}^{Y^{\prime}}(t):=\left(\exp \left[-i \frac{h}{2} V_{N}\right] \exp \left[-i h H_{0, N}\right] \exp \left[-i \frac{h}{2} V_{N}\right]\right)^{n} v_{N} .
$$

We may thereby conclude that the $h$-dependence provided by these numerical results is in very good agreement with relations (67) and (68) of the corollary stated at the very end of Section 2, and with relation (69) as well, which are all valid in the autonomous case. It is, therefore, deemed appropriate to compute the solution to (71)-(73) by means of the product approximations (34), (35) and (90), with a very good rate of convergence in each case and with stability and accuracy of the numerical results, which we have also compared with those obtained by using MatLab's ode45.

(2) The crucial fact that all our approximating operators are unitary with respect to the supremum-norm is also visible numerically. Whereas the $L^{2}(\mathbb{R}, \mathbb{C})$ norm of the solution is indeed essentially constant in time in all cases, up to floating point machine errors, this is not so much the case for the results we got through MatLab's ode45 as we shall see further below in Figure 14.

We now describe the results which pertain to the computation of the solution to $(30)$, that is,

$$
\begin{aligned}
i \frac{\partial u(x, t)}{\partial t} & =H(t) u(x, t), \quad(x, t) \in \mathbb{R} \times(0, T], \\
u(x, 0) & =v(x), \quad x \in \mathbb{R}
\end{aligned}
$$

where

$$
H(t):=-\frac{1}{2} \frac{\partial^{2}}{\partial x^{2}}+\frac{1}{2} x^{2}+\lambda(1-\sin \omega t) x^{4},
$$

and where we choose once again the initial condition $v$ to be the solution to (73) computed with MatLab's eigs. As before we compute the exponentials by means of MatLab's expm, which we apply this time to the difference between

$$
u_{N, n}(t):=\prod_{\gamma=n-1}^{0} \exp \left[-i h H_{N}(\gamma h)\right] v_{N}
$$

and (26), (27) and (28), respectively, where $H_{N}$ denotes the restriction of (91) to the Faedo-Galerkin subspace we defined in Section 2. This is indeed justified in that (92) provides a reference approximation for the solution to (30) according to the first equality in (65) taken with $s=0$. We display the corresponding root mean square errors as a function of the uniform step size $h$ in the figures below for $N=25$ and various values of $\lambda, t$ and $\omega$. In so doing we note one important difference between the results shown and those of Figure 7, to wit, the fact that the root mean square error between (92) and (27) on the one hand, and between (92) and (28) on the other hand, no longer coincide. Moreover, we choose $h^{*}=10^{-5}$ as the reference step size to compute (92), a much smaller 
value than that used for the computation of (26), (27) and (28), the reason being that the operators $H_{N}(\gamma h)$ taken at difference time steps do not commute:

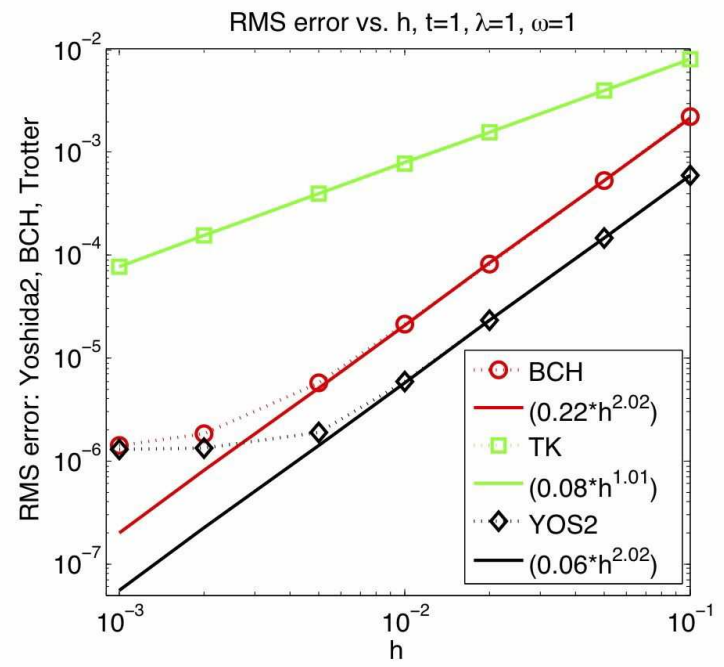

Figure 8: comparisons of the RMS errors of the various splittings with (92) for the non-autonomous problem, for $N=25, \lambda=1, t=1$ and $\omega=1$. Error data for $h<5 \times 10^{-3}$ are not used in the fits because their values are smaller than the reference error.

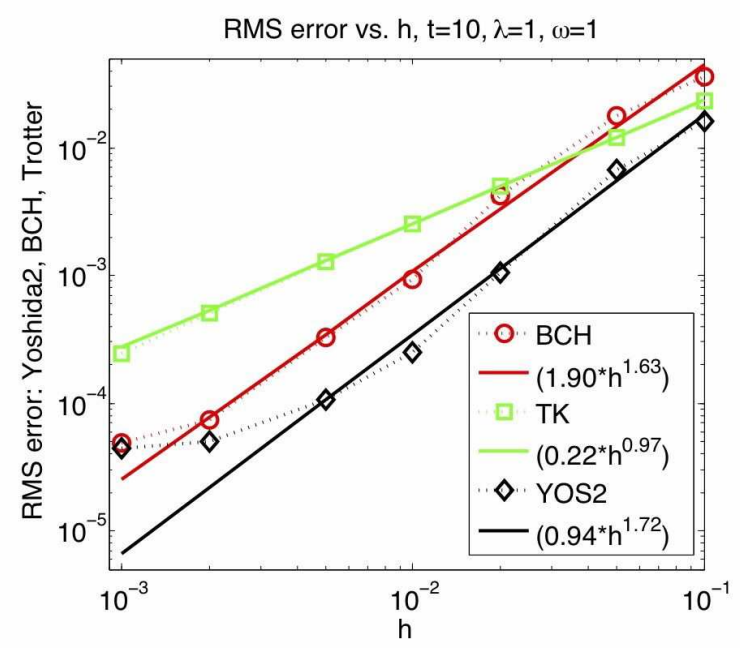

Figure 9: same comparisons and restrictions as in Figure 8 but with $N=25$, $\lambda=1, t=10$ and $\omega=1$. 


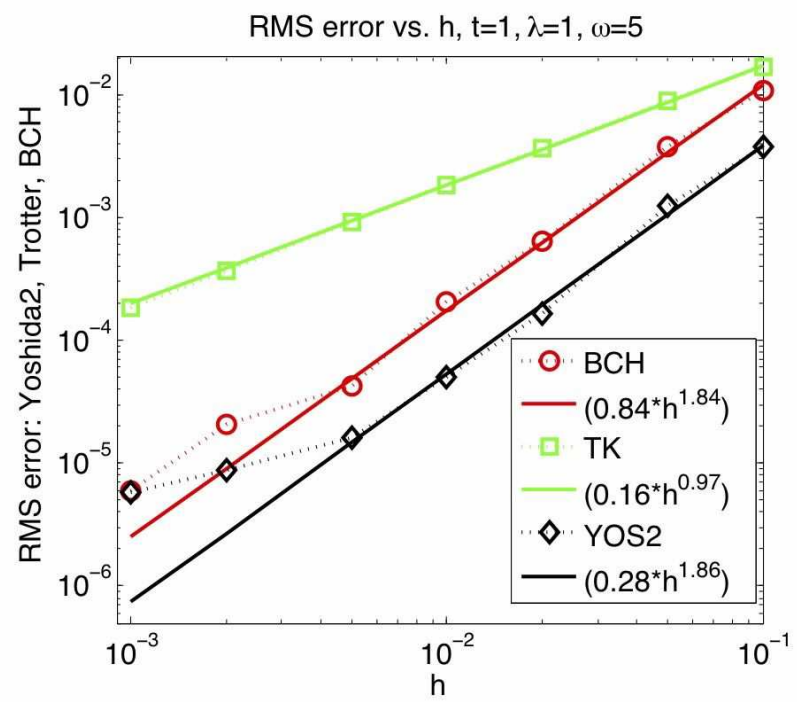

Figure 10: same comparisons and restrictions as in the above figures but with $N=25, \lambda=1, t=1$ and $\omega=5$.

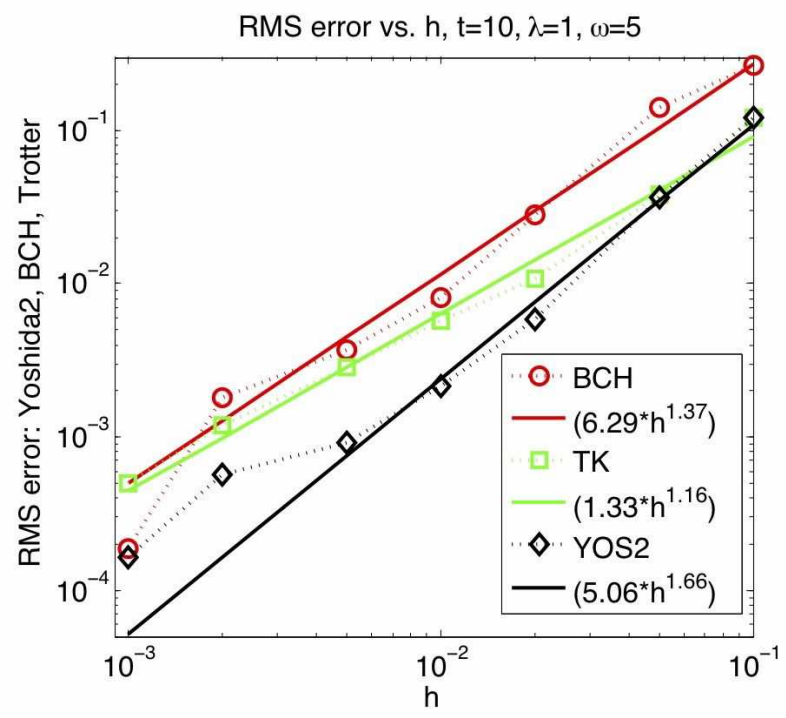

Figure 11: same comparisons and restrictions as in the above figures but with $N=25, \lambda=1, t=10$ and $\omega=5$. 


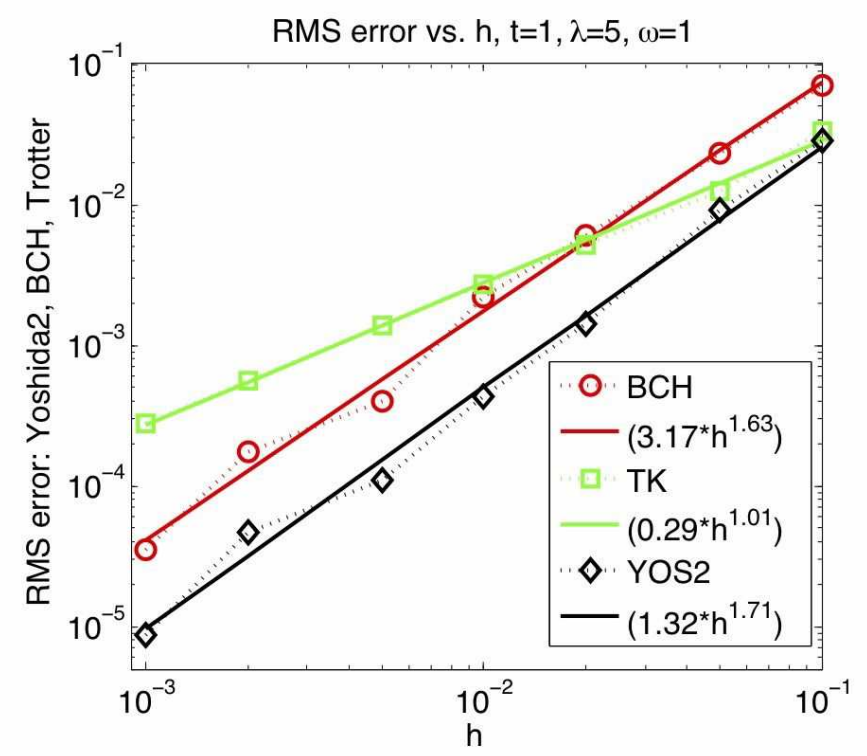

Figure 12: same comparisons and restrictions as in the above figures but with $N=25, \lambda=5, t=1$ and $\omega=1$.

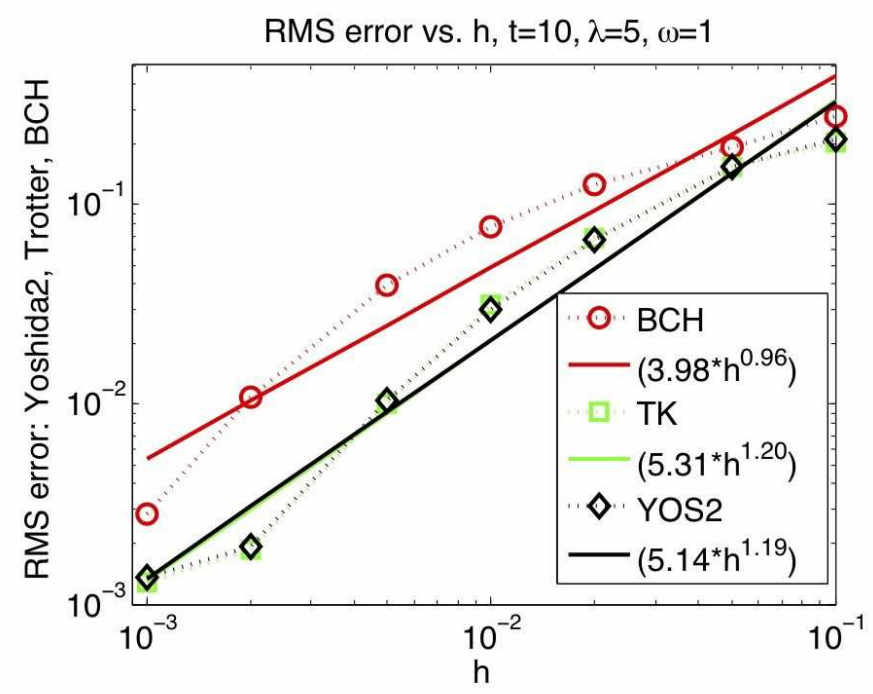

Figure 13: same comparisons and restrictions as in the above figures but with $N=25, \lambda=5, t=10$ and $\omega=1$.

Thus, we may conclude that (26), (27) and (28) provide very good stable and accurate algorithms for the computation of the solution to (30), in spite of the fact that the respective rates of convergence seem to be numerically sensitive 
to the values of $\lambda, t$ and $\omega$, thereby making them somewhat different from the analytical predictions (66), (67) and (68).

We have also compared the above results with that obtained by means of the quite robust and reliable integrator MatLab's ode45, which, however, does not keep the $L^{2}(\mathbb{R}, \mathbb{C})$-norm of the solution to (30) constant in time as much as the product approximations do, as shown in the following figure:

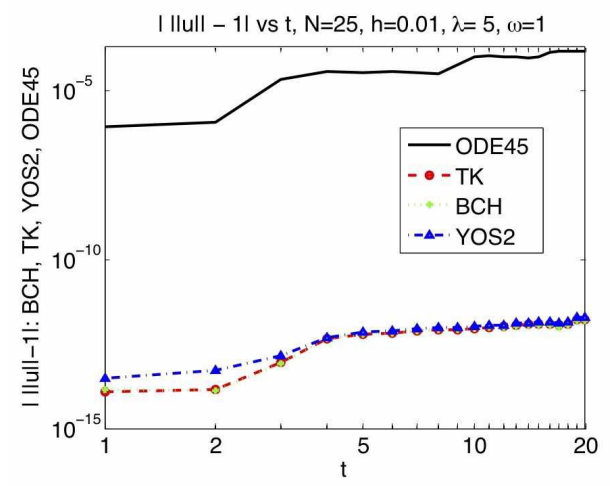

Figure 14: time evolution of the $L^{2}$-norm of the solution to (30) as computed with the product approximations and with MatLab's ode45, for $N=25$,

$$
h=10^{-2}, \lambda=5 \text { and } \omega=1 .
$$

Finally, we provide a last piece of information regarding (30) by plotting this time the root mean square errors we computed above as a function of $t$, which shows that at least for relatively small times those root mean square errors are increasing:

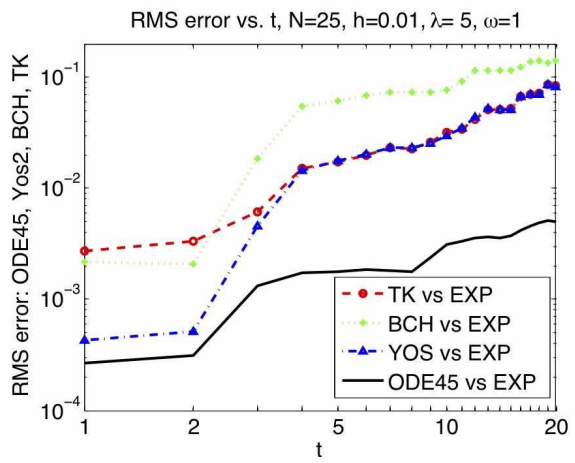

Figure 15: comparison of the RMS errors of the various splittings and MatLab's ode45 with (92) labeled as EXP for the non-autonomous problem, for $N=25, h=10^{-2}, \lambda=5$ and $\omega=1$.

Concluding REMARK AND PROVEnANCE INFORMation. The preceding results complete our analytical and numerical investigations of Problems (2) and (30), thus showing that the product approximations of Section 1 are rigorously 
correct within the framework of the Faedo-Galerkin scheme we defined in Section 2 , and numerically verified with high accuracy in this section. This suggests that those product approximations might remain valid in the limit $N \rightarrow+\infty$, and for more general non-autonomous quantum systems as well, but rigorous proofs of such statements are clearly lacking at the present time.

All the simulations in this paper were run on an Apple Macbook using MatLab version R2012a. The processor was an Intel Core 2 Duo, and the Mac operating system was Mac OS-X, version 10.6.8. Any interested reader is welcome to the MatLab code.

Acknowledgements. Both authors are particularly indebted to Professors T. Rivière and C. Schwab for having made the visit of the second author in Zurich financially possible through funds from the Forschungsinstitut für Mathematik and the Seminar für Angewandte Mathematik of the ETHZ, the warm hospitality of which they gratefully acknowledge.

\section{Appendix: A Formal WKB Approximation}

In this section we consider anew the equation

$$
\left(-\frac{1}{2} \frac{d^{2}}{d x^{2}}+\frac{1}{2} x^{2}+\lambda x^{4}\right) v(x)=E_{0} v(x), \quad x \in \mathbb{R}
$$

and obtain yet other approximations for $E_{0}$ and $v$. Our method in this appendix is essentially formal, for our focus is not so much on mathematical rigor as it is on the success of the numerical algorithm we shall be using. Since (93) is an eigenvalue problem, $E_{0}$ and $v$ should in fact be computed simultaneously. However we proceed somewhat differently by first choosing $E_{0}=E_{0}^{*}$ in (93), where $E_{0}^{*}$ is given by (77) or (79). By analogy with (74) which satisfies (93) exactly when $\lambda=0$ and $E_{0}=\frac{1}{2}$, or with the best Gaussian approximation (81), we then look for an approximate solution to (93) of the form

$$
v(x)=e^{-w(x)}
$$

where $w: \mathbb{R} \mapsto \mathbb{R}^{+}$is sufficiently smooth and even. The differential equation for this function being

$$
w^{\prime \prime}(x)-w^{\prime}(x)^{2}=2 E_{0}-x^{2}-2 \lambda x^{4},
$$

this amounts to finding an approximate solution to (95). Let $x_{0}$ be the only positive root to

$$
2 \lambda x^{4}+x^{2}-2 E_{0}=0 .
$$

For $|x| \leq x_{0}$ where

$$
2 E_{0}-x^{2}-2 \lambda x^{4} \geq 0
$$

let us consider

$$
w_{<}(x):=w_{2} x^{2}+w_{4} x^{4}+w_{6} x^{6} .
$$


Substituting (96) into (95) and neglecting all powers higher than four in $\left(w^{\prime}\right)^{2}$ we obtain

$$
w_{<}(x)=E_{0} x^{2}+\frac{1}{12}\left(4 E_{0}^{2}-1\right) x^{4}+\frac{1}{15}\left(\frac{2 E_{0}}{3}\left(4 E_{0}^{2}-1\right)-\lambda\right) x^{6} .
$$

We note that for $\lambda=0$ and $E_{0}=\frac{1}{2}$, the preceding expression satisfies (95) exactly. Now for $|x| \geq x_{0}$ where

$$
2 E_{0}-x^{2}-2 \lambda x^{4} \leq 0
$$

let us consider

$$
w_{>}(x):=w_{<}\left(x_{0}\right)+\int_{x_{0}}^{|x|} d y\left(2 \lambda y^{4}+y^{2}-2 E_{0}\right)^{\frac{1}{2}} .
$$

For each $x \in \mathbb{R}$ we then define the function

$$
w_{\text {шкв }}(x):=\chi(x) w_{<}(x)+(1-\chi(x)) w_{>}(x)
$$

with

$$
\chi(x)=\left\{\begin{array}{c}
1 \text { for }|x| \leq x_{0}, \\
e^{-\frac{\left(|x|-x_{0}\right)^{2}}{16 x_{0}^{2}}} \quad \text { for }|x| \geq x_{0} .
\end{array}\right.
$$

It is clear that our method to obtain (98) bears some analogy with the classical WKB method (see, for instance, [3]), and it turns out that (94) with $w=w_{\text {шкв }}$ given by (98) provides a very good numerical approximation for the solution to (93). In order to see this we first express the second term on the right-hand side of (97) in terms of elliptic integrals, which is possible by means of the identity

$$
\lambda y^{4}+\frac{y^{2}}{2}-E_{0}=\lambda\left(y^{2}+\mu\right)\left(y^{2}-\nu\right)
$$

where

$$
\mu:=\frac{1}{4 \lambda}\left(\left(1+16 \lambda E_{0}\right)^{\frac{1}{2}}+1\right)>0
$$

and

$$
\nu:=\frac{1}{4 \lambda}\left(\left(1+16 \lambda E_{0}\right)^{\frac{1}{2}}-1\right)=x_{0}^{2}>0 .
$$

Indeed from (97), (99) and Relation 3-155-6 in Chapter 3 of [15] we obtain

$$
\begin{aligned}
w_{>}(x)= & w_{<}\left(x_{0}\right)+\sqrt{2 \lambda} \int_{x_{0}}^{|x|} d y\left(\left(y^{2}+\mu\right)\left(y^{2}-\nu\right)\right)^{\frac{1}{2}} \\
= & w_{<}\left(x_{0}\right)+\frac{1}{3}(2 \lambda(\mu+\nu))^{\frac{1}{2}}((\nu-\mu) E(\phi, k)-\nu F(\phi, k)) \\
& +\sqrt{2 \lambda} \frac{x^{2}+\mu-\nu}{3|x|}\left(\left(x^{2}+\mu\right)\left(x^{2}-\nu\right)\right)^{\frac{1}{2}},
\end{aligned}
$$


where $E(\phi, k)$ and $F(\phi, k)$ are elliptic integrals of the second and first kind, respectively, with amplitude

$$
\phi=\arccos (\sqrt{\nu} /|x|)
$$

and modulus

$$
k=\left(\frac{\mu}{\mu+\nu}\right)^{\frac{1}{2}} .
$$

The interest in (100) now lies in the fact that Landen's transformation (see, for instance, [1]) provides an algorithm for the simultaneous computation of $E(\phi, k)$ and $F(\phi, k)$ in terms of the complete elliptic integrals

$$
E=E\left(\frac{\pi}{2}, k\right)
$$

and

$$
K=F\left(\frac{\pi}{2}, k\right),
$$

respectively, where (101) and (102) can be computed by means of two polynomials $P$ and $Q$ in the variable $z=1-k^{2}$ through the relation $P(z)-Q(z) \ln z$ according to [22]. Indeed we can first initialize a set of $m$ values

$$
\mathbf{x}:=\left(x_{1}, \ldots, x_{m}\right) \mapsto \phi:=\left(\phi_{1}, \ldots, \phi_{m}\right)
$$

where

$$
\phi_{j}=\arccos \left(\sqrt{\nu} / x_{j}\right)
$$

for each $j \in\{1, \ldots, m\}$, by setting

$$
\mathbf{t}=\tan (\phi), a=1, b=\sqrt{1-k^{2}}, c=k, d=1, \mathbf{m}_{\mathbf{0}}=\mathbf{0}, \mathbf{e}_{\mathbf{s}}=\mathbf{0} .
$$

According to [22] we then follow the descending Landen series on the triplet $(a, b, c)$ until $|c / a|$ is smaller than the relative machine precision which is about $2.2 \times 10^{-16}$ (see, for instance, [17]), with quadratic convergence in five to six steps according to [5] or [6]. In this process it is important that the arithmeticgeometric mean sequence depends only upon $k$ and not on $\phi$, which allows the simultaneous computation of $\phi$-vectors and thereby multiple computations of elliptic functions which can be easily parallelized. Indeed parallel versions of the tangent-, arctangent- and sine functions do exist in the software library (see, for instance [17] and [23]). We illustrate the algorithm in the diagram below. The result of that simultaneous computation of $E(\phi, k)$ and $F(\phi, k)$ together with (100) then leads to the computation of (98), and thereby to that of (94) with $w=w_{\text {WKB }}$, which we denote by $v_{\mathrm{E}}$. In Figures 16 and 17 we compare $v_{\mathrm{E}}$ with $v$ computed with MatLab's eigs, with the best Gaussian approximation $v_{\alpha^{*}}$, and with yet another approximation $v_{\mathrm{Q}}$ to (94) obtained from (98) by means of MatLab's quad, which we use as a control. Finally, the numerical integration of

$$
\begin{aligned}
E_{0, \mathrm{wKB}} & :=\left(H v_{\mathrm{wKB}}, v_{\mathrm{wKB}}\right)_{2} \\
= & \int_{\mathbb{R}} d x\left(\frac{1}{2}\left|v_{\mathrm{wKB}}^{\prime}(x)\right|^{2}+\frac{1}{2} x^{2}\left|v_{\mathrm{wKB}}(x)\right|^{2}+\lambda x^{4}\left|v_{\mathrm{wKB}}(x)\right|^{2}\right)
\end{aligned}
$$


where

$$
v_{\text {WKB }}:=e^{-w_{\text {WKB }}}
$$

provides yet another approximation for the lowest eigenvalue in (93), which we compare with $E_{0}$ computed with MatLab's eigs and with the Gaussian approximation $E_{0}^{*}$ in Figure 18. In the following diagram which illustrates our algorithm we set $n_{\pi / 2}=\left\lfloor\phi /\left(\frac{\pi}{2}\right)\right\rfloor$ and, in the fourth step in the loop, we mean

$$
t_{j} \longleftarrow \frac{(1+r) t_{j}}{1-r t_{j}^{2}}
$$

for every $j \in\{1, \ldots, m\}$ :

$$
\begin{aligned}
& \text { while }(|c / a|>\epsilon) \\
&\{ \leftarrow \frac{b}{a} \\
& \phi \leftarrow \phi+\arctan (r \mathbf{t})+\pi \mathbf{m}_{0} \\
& \mathbf{m}_{0} \leftarrow\left\lfloor\frac{\phi+\pi / 2}{\pi}\right\rfloor \\
& \mathbf{t} \leftarrow \frac{(1+r) \mathbf{t}}{1-r t^{2}} \\
& c \leftarrow \frac{a-b}{2} \\
& s \leftarrow \frac{\sqrt{a b}}{2} \\
& a \leftarrow s \\
& b \leftarrow \mathbf{e}_{s}+c \sin (\phi) \\
& d \mathbf{e}_{s} \\
&\} \quad a d \\
& \mathbf{t}_{3} \leftarrow \frac{\arctan (\mathbf{t})+\pi \mathbf{m}_{0}}{\mathbf{t}_{2} \leftarrow \frac{\mathbf{t}_{3}}{K}+\mathbf{e}_{s}+n_{\pi / 2} E, \quad \mathbf{t}_{1} \leftarrow \mathbf{t}_{3}+n_{\pi / 2} K} \\
& F(\phi, k)=\mathbf{t}_{1}, E(\phi, k)=\mathbf{t}_{2}
\end{aligned}
$$

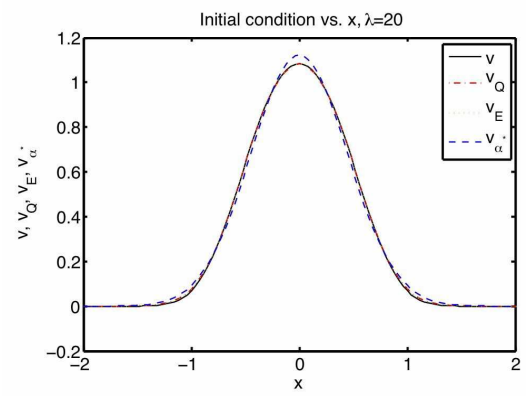

Figure 16: comparison of $v$ computed with MatLab's eigs with $v_{\alpha^{*}}$ and the two computed variants $v_{\mathrm{E}}$ and $v_{\mathrm{Q}}$ of $v_{\mathrm{WKB}}$, for $\lambda=20$. 


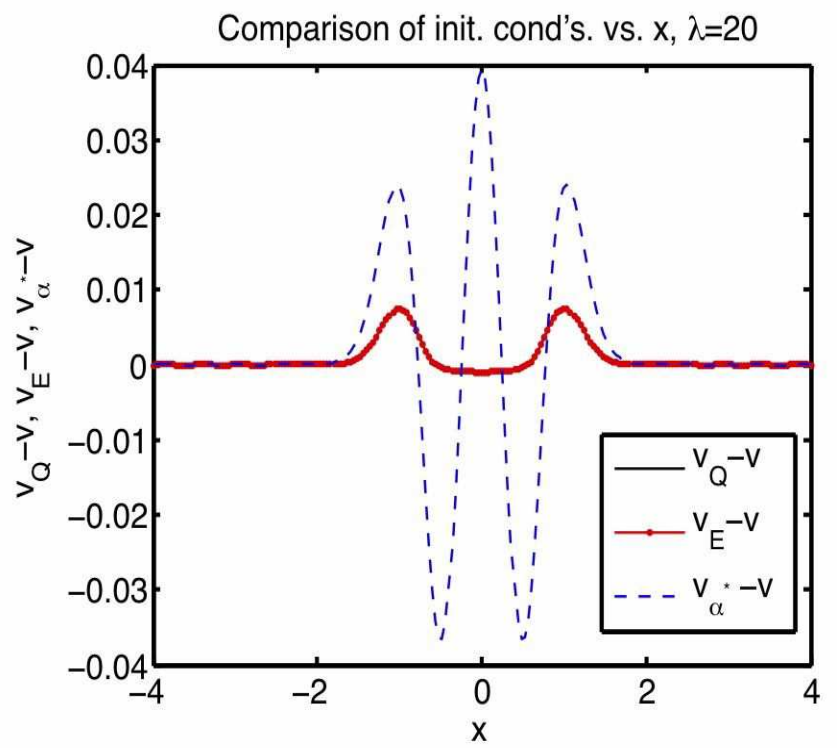

Figure 17: comparison of the differences $v_{\mathrm{Q}}-v, v_{\mathrm{E}}-v$ and $v_{\alpha^{*}}-v$ of the functions defined in the preceding figure, for $\lambda=20$.

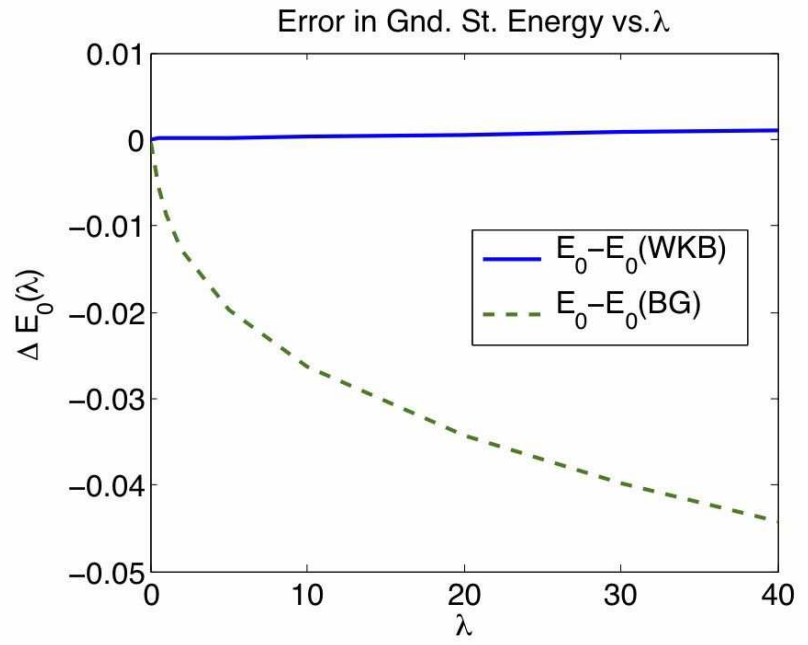

Figure 18: comparison of $E_{0, \mathrm{WKB}}$ and $E_{0, \mathrm{BG}}:=E_{0}^{*}$ with $E_{0}$ computed by means of MatLab's eigs: $E_{0, \text { WKB }}$ provides yet an improved value for the lowest eigenvalue in (93). 


\section{References}

[1] Abramowitz, M., Stegun, I. A., Handbook of Mathematical Functions, Dover Publications, New York (1972).

[2] Apostol, T. M., Calculus, II: Multi-Variable Calculus and Linear Algebra, with Applications to Differential Equations and Probability, John Wiley and Sons, New York (1969).

[3] Bender, C. M., Orszag, S. A., Advanced Mathematical Methods for Scientists and Engineers. Asymptotic Methods and Perturbation Theory, Springer, New York (1999).

[4] Bender, C. M., Wu, T. T., Anharmonic Oscillator, The Physical Review 184, 5 (1969) 1231-1260.

[5] Borwein, J. M., Borwein P. B., Pi and the AGM: A Study in Analytical Number Theory and Computational Complexity, Wiley-Interscience Publication, John Wiley, New York (1987).

[6] Carlson, B. C., Algorithms Involving Arithmetic and Geometric Means, The American Mathematical Monthly 78 (1971) 496-505.

[7] Chernoff, P. R., Note on Product Formulas for Operator Semigroups, Journal of Functional Analysis 2 (1968) 238-242.

[8] Chernoff, P. R., Product Formulas, Nonlinear Semigroups, and Addition of Unbounded Operators, Memoirs of the American Mathematical Society 140, Providence (1974).

[9] Chorin, A. J., Hughes, T. J. R., McCracken, M. F., Marsden, J. E., Product Formulas and Numerical Algorithms, Communications on Pure and Applied Mathematics 31 (1978) 205-256.

[10] Davies, E. B., One-Parameter Semigroups, Academic Press, London (1980).

[11] Dormand, J. R., Prince, P. J., Practical Runge-Kutta Processes, SIAM Journal on Scientific and Statistical Computing 10 (1989) 977-989.

[12] Exner, P., Neidhardt, H., Zagrebnov, V. A., Remarks on the Trotter-Kato Product Formula for Unitary Groups, Integral Equations and Operator Theory 69 (2011) 451-478.

[13] Faris, W. G., The Product Formula for Semigroups defined by Friedrichs Extensions, Pacific Journal of Mathematics 22 (1967) 47-70.

[14] Faris, W. G., Product Formulas for Perturbations of Linear Propagators, Journal of Functional Analysis 1 (1967) 93-108. 
[15] Gradshteyn, I. S., Ryzhik, I. M., Table of Integrals, Series and Products, corrected and enlarged edition, Academic Press, New York (1980).

[16] Hairer, E., Lubich, C., Wanner, G., Geometric Numerical Integration: Structure-Preserving Algorithms for Ordinary Differential Equations, Springer, New York (2006).

[17] Higham, D. J., Higham, N. J., Mathlab Guide, Society for Industrial and Applied Mathematics, Philadelphia (2005).

[18] Higham, N. J., The Scaling and Squaring Method for the Matrix Exponential Revisited, SIAM Journal on Matrix Analysis and Applications 26 (2005) 1179-1193.

[19] Jahnke, T., Lubich, C., Error Bounds for Exponential Operator Splittings, BIT Numerical Mathematics 40 (2000) 735-744.

[20] Johnson, G. W., Lapidus, M. L., The Feynman Integral and Feynman's Operational Calculus, Oxford University Press, Oxford (2000).

[21] Moler, C., Van Loan C., Nineteen Dubious Ways to Compute the Exponential of a Matrix, Twenty-Five Years Later, SIAM Review 45 (2003) 3-49.

[22] Moshier, S. L. B., Methods and Programs for Mathematical Functions, Ellis Horwood Series in Mathematics and its Applications, John-Wiley, New York (1989), http://www.moshier.net/\#Cephes

[23] Petersen, W. P., Arbenz, P., Introduction to Parallel Computing, Oxford University Press, Oxford (2004).

[24] Reed, M., Simon, B., Methods of Modern Mathematical Physics, II: Fourier Analysis, Self-Adjointness, Academic Press, New York (1975).

[25] Trotter, H., On the Product of Semigroups of Operators, Proceedings of the American Mathematical Society 10 (1959) 545-551.

[26] Vuillermot, P.-A., A Generalization of Chernoff's Product Formula for Time-Dependent Operators, Journal of Functional Analysis 259 (2010) 2923-2938.

[27] Vuillermot, P.-A., Wreszinski, W. F., Product Approximations for Solutions to a Class of Evolution Equations in Hilbert Space, Portugaliae Mathematica 68 (2011) 317-343.

[28] Vulllermot, P.-A., Wreszinski, W. F., Zagrebnov, V. A., A General Trotter-Kato Formula for a Class of Evolution Operators, Journal of Functional Analysis 257 (2009) 2246-2290.

[29] Yoshida, H., Construction of Higher-Order Symplectic Integrators, Physics Letters A 150 (1990) 262-268. 